$\mathrm{IC} / 97 / 198$

hep-th/9712047

\title{
Aspects of U-Duality in Matrix Theory
}

\author{
Matthias Blau and Martin O'Loughlin \\ ICTP \\ Strada Costiera 11, 34014 Trieste \\ Italy
}

\begin{abstract}
We explore various aspects of implementing the full M-theory U-duality group $E_{d+1}$, and thus Lorentz invariance, in the finite $N$ matrix theory (DLCQ of M-theory) describing toroidal IIA-compactifications on $d$ tori: (1) We generalize the analysis of Elitzur et al. (hep-th/9707217) from $E_{d}$ to $E_{d+1}$ and identify the highest weight states unifying the momentum and flux $E_{d}$-multiplets into one $E_{d+1}$-orbit. (2) We identify the new symmetries, in particular the Weyl group symmetry associated to the $(d+1)$ 'th node of the $E_{d+1}$ Dynkin diagram, with Nahm-duality-like symmetries (N-duality) exchanging the rank $N$ of the matrix theory gauge group with other (electric, magnetic, ...) quantum numbers. (3) We describe the action of N-duality on BPS bound states, thus making testable predictions for the Lorentz invariance of matrix theory. (4) We discuss the problems that arise in the matrix theory limit for BPS states with no top-dimensional branes, i.e. configurations with $N=0$. (5) We show that $\mathrm{N}$-duality maps the matrix theory SYM picture to the matrix string picture and argue that, for $d$ even, the latter should be thought of as an M-theory membrane description (which appears to be well defined even for $d>5$ ). (6) We find a compact and unified expression for a U-duality invariant of $E_{d+1}$ for all $d$ and show that in $d=5,6$ it reduces to the black hole entropy cubic $E_{6^{-}}$and quartic $E_{7}$-invariants respectively. (7) Finally, we describe some of the solitonic states in $d=6,7$ and give an example (a 'rolled-up' Taub-NUT 6-brane) of a configuration exhibiting the unusual $1 / g_{s}^{3}$-behaviour.
\end{abstract}

\footnotetext{
${ }^{1}$ e-mail: mblau,mjol@ictp.trieste.it
} 


\section{Contents}

$\begin{array}{lll}1 & \text { Introduction } & 1\end{array}$

2 U-Duality and Matrix Theory 5

2.1 M-Theory Facts . . . . . . . . . . . . . . . . . . . 5

2.2 The T-dual SYM-like Picture . . . . . . . . . . . . . . . . 7

2.3 The Matrix Theory Limit and the DLCQ of M-Theory . . . . . . . . 11

2.4 D $d$-Brane Backgrounds and Bound States $\ldots \ldots \ldots$

3 Extending the U-Duality Group from $E_{d}$ to $E_{d+1}$ : The Algebra 16

3.1 The Algebraic Set-Up $\ldots \ldots \ldots \ldots \ldots . \ldots \ldots$

3.2 The Weyl Group of $E_{d+1} \ldots \ldots \ldots \ldots \ldots \ldots$

3.3 Fundamental Weights of $E_{d+1} \ldots \ldots \ldots \ldots \ldots$

3.4 The Highest Weights of the U-Duality Group $E_{d+1} \ldots \ldots$. . . . . 21

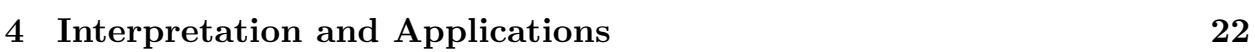

4.1 U-Duality, Nahm Duality, and the Matrix Theory Limit . . . . . . . 23

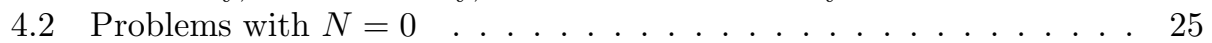

4.3 N-Duality, (1+1)-Dimensional Backgrounds and Matrix Strings . . . 28

\begin{tabular}{lll|ll}
\hline 4.4 & An M2-brane Picture of Matrix Theory for $d$ Even & $\ldots$ & $\ldots$
\end{tabular}

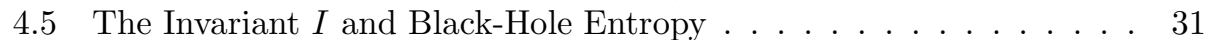

$4.6 \quad$ A Look at Some States in $d=6$ and $d=7 \ldots \ldots \ldots$. . . . . . . 32

$4.7 \quad$ A Rolled-Up Taub-NUT Soliton and $1 / g_{s}^{3}$-States $\ldots . . . . . . . .35$

\begin{tabular}{ll}
\hline References & 37
\end{tabular}

\section{INTRODUCTION}

It is well known that the equations of motion of eleven-dimensional supergravity compactified on a $(d+1)$-torus exhibit a hidden non-compact global symmetry group $E_{d+1(d+1)}$.1 Once the massive modes of string theory are included, this full structure does not survive. However, in [1] it was conjectured that a discrete subgroup $E_{d+1(d+1)}(\mathbb{Z})$ survives in the full string theory, in particular via its action on the BPS spectrum and as a discrete set of identifications on the supergravity moduli space. This group, known as the U-duality group, has played a central role in the subsequent investigations of string theory dualities.

In a recent paper [2], the action of the Weyl group of the corresponding $E_{d} \subset E_{d+1}$ upon states arising from wrapped M-branes was discussed. These

\footnotetext{
${ }^{1} E_{3(3)}=S L(3) \times S L(2), E_{4(4)}=S L(5), E_{5(5)}=S O(5,5)$
} 
results taken together with the dictionary from M-theory to the super-YangMills (SYM) variables of matrix theory [3] enable one to discuss the action of U-duality in the SYM variables, and hence on the BPS spectrum of matrix theory. An excellent and authoritative review of matrix theory is now available [4, and we refer to it for an extensive list of references to earlier work on various aspects of matrix theory.

As the eleventh direction of M-theory plays a distinguished role in the transition from M-theory to its matrix theory description, it at first does not appear straightforward to extend this to an action of the full duality group $E_{d+1}$. Here we will propose such an extension, based once again on the Mtheory dictionary as well as on recent observations by Sen and Seiberg [5, 6] connecting the matrix theory limit of M-theory to the discrete light-cone quantization (DLCQ) of M-theory proposed by Susskind [7].

A small but crucial difference between our approach and that of [2] is that we consider the action of the Weyl group on the momenta of the BPS states, rather than on the energies. This turns out to be essential in the SYM picture of compactified matrix theory, if one wishes to combine the momentum and flux multiplets into a single multiplet of the full U-duality group.

The issue of finding the matrix theory realization of this full U-duality symmetry group of M-theory is, of course, closely related to the issue of rotational (Lorentz) invariance of matrix theory, in particular to the ability to change the value of the longitudinal or light-cone momentum. While we will not be able to resolve this issue, we indeed find a new duality symmetry in $E_{d+1}$, associated with the $(d+1)$ 'th node of the Dynkin diagram of $E_{d+1}$, exchanging the rank (light-cone momentum) $N$ of the gauge group with quanta of flux. Both for this reason and because this duality is reminiscent of Nahm duality [8], we will refer to this as N-duality. The necessity to discuss all the light-cone sectors simultaneously in a more complete formulation of the theory had been anticipated by Susskind [7]. A realization of N-duality symmetry in such a theory would be an indication of its Lorentz invariance. Nahm duality has recently been discussed in a closely related context (SYM in $(3+1)$ dimensions) in [9].

In trying to interpret the action of this extended U-duality group as an action on the BPS states of a SYM theory in, say, $(3+1)$ dimensions, we encounter the problem that a state with non-zero $N$ can be mapped to a state with $N=0$. Clearly this is a rather singular state from a SYM point of view and indeed we find that the masses of such states diverge in the matrix theory limit. In $(4+1)$ dimensions, such states correspond to systems of two-branes and zero-branes on a four-torus $T^{4}$ (the absence 
of wrapped four-branes implying $N=0$ ), and such configurations can be interpreted by generalizing the notion of a vector bundle to that of a sheaf (see the discussion in [10, section 5.3]).

In the case $d=3$, on the other hand, and in most other cases, such an interpretation is not available and some new idea appears to be required. While we were in the final stages of writing this paper and trying to come to terms with this problem, papers by Connes, Douglas, and Schwarz [11] and Douglas and Hull [12] appeared which (if we understand them correctly) seem to address this issue. In these papers it is argued that in order to see the full U-duality group one needs to consider SYM theory on a non-commutative torus (see the references in [11, 12]). Indeed, [11, eq. (5.14)] shows that the non-commutativity of the torus 'regularizes' the infinite masses arising for $N=0$ in the commutative case. We will come back to this issue in section 4.2 of this paper.

This paper is organized as follows. In section 2 we set up the framework for the discussion of $E_{d+1}$-duality in matrix theory. We first describe the emergence of (the Weyl group of) $E_{d+1}$ on the M-theory side by an algebraic construction inspired by [2] and then describe the action of this U-duality group on the T-dual II side. In particular we describe the N-duality transformation in terms of its action on the parameters of the $\widetilde{I I}$ theory and identify it as the transformation $T^{d-1} S_{I I B} T^{d-1}$ where $T^{d-1}$ denotes a T-duality on $(d-1)$ of the circles of the $d$-torus and $S_{I I B}$ is the S-duality of the type IIB string theory. We then briefly review the matrix theory limit of the II theory, as described in [5, 6] as well as the BPS mass formula for threshold and non-threshold bound-states.

In section 3, we generalize (and modify in some respects) the algebraic analysis of [2]. We realize the duality symmetries described in section 2.2 as Weyl reflections in the root space of $E_{d+1}$, determine the fundamental weights of $E_{d+1}$ in terms of the $\widetilde{\text { II }}$ parameters and indentify the highest weights unifying the momentum and flux $E_{d}$-multiplets into a single $E_{d+1}$-multiplet. In particular, for $d=8$ we determine the $E_{9}$-representation that arises to be the unique integrable representation of affine $E_{8}$ at level one, and for $d=9$ we find that the representation of the hyperbolic Lie algebra $E_{10}$ is the one associated with the null-root of $E_{9}$.

In section 4 we discuss various consequences and applications of this extended U-duality group. We exhibit more explicitly the action of N-duality as a Nahm duality on the quantum numbers (and thus BPS states) of the matrix theory and we point out the difficulties with the $N=0$ states mentioned above. We also discuss the (less problematic as mass preserving) 
active interpreation of U-duality as an action providing different pictures of the same state in different string theories. In particular, we point out that $\mathrm{N}$-duality always provides an effectively $(1+1)$-dimensional SYM description of any bound-state involving background $\mathrm{D} d$-branes (or D0-branes on the M-theory/IIA side) and that this reproduces the matrix string dictionary of [13]. We will also argue that in even dimensions the matrix string picture is perhaps better thought of as an M-theory membrane theory, and this appears to be well defined even for $d>5$.

In the algebraic analysis of section 3 , an important role is played by a Uduality invariant $I$ for which we have a compact and unified expression for any $d$. In section 4.5 we will show explicitly that for $d=5,6$ this reduces to the cubic invariant of $E_{6}$ and quartic invariant of $E_{7}$ respectively which appear in the analysis of black hole entropies in five and four dimensions. Finally, we return to the $E_{d+1}$ multiplets of single-particle states determined in section 3 . In $d \leq 5$ only one new state (the highest weight state, an $E_{d^{-}}$ singlet) is required to unify the momentum and flux states. For $d>5$, however, we find other new states in the Weyl group orbit of $E_{d+1}$, one for $d=6,57$ for $d=7$, etc. These have no obvious SYM interpretation and should be indicative of the new physics associated with lower-dimensional compactifications of string theory. We discuss some of the (old and new) states in $d=6,7$ and identify concretely some of the solitonic objects responsible for the 'strange' states $\left(\sim 1 / g_{s}^{3}\right)$ found in [2]. In particular, we find that - somewhat unexpectedly - the periodic Taub-NUT soliton encountered in the context of corrections to the hypermultiplet moduli space in the vicinity of the conifold singularity [14 makes an appearance here.

In a recent paper [15, Hull has announced that he has also obtained the generalization of [2] from $E_{d}$ to $E_{d+1}$ [16].

A final remark on terminology and a caveat: Even though, strictly speaking, for $d>3$ SYM theory itself is only an adequate description of the matrix theory limit of a given toroidal compactification at long wavelengths, we will find it convenient to use a SYM-like terminology when e.g. discussing boundstates and the action of the U-duality group on the quantum numbers. We will therefore also occasionally refer to the matrix theory limit as a 'SYM' or SYM-like theory.

The caveat regards the fact that matrix theories are known only for $d \leq 5$ [17, 6, 5, 我. Already for $d=6$ there are problems, and for $d \geq 7$ the situation gets worse as the background configurations of such branes are severely restricted. Nevertheless, provided that matrix theories for $d>5$ exist and reduce at low energies to SYM, the considerations of this paper 
regarding U-duality orbits of BPS states should, in the same spirit as in [15], be valid and provide strong constraints on the matrix theories themselves.

\section{U-Duality And Matrix TheOry}

\subsection{M-ThEORY FACTS}

Upon compactification of M-theory on a rectangular $(d+1)$-torus $T^{d+1}$, the parameters of M-theory are $\ell_{P}, R_{i}, i=1 \ldots d$, and $R_{11}$. When $R_{11}<<R_{i}$ this is simply the type IIA string on $T^{d}$. The IIA string theory is mapped to itself under T-duality on two different circles $\left(x_{i}, x_{j}\right)$, and the new IIA theory will in general have a different string coupling $g_{s}$. Using the relationship between M-theory and IIA variables, $R_{11}=l_{s} g_{s}$ and $\ell_{P}^{3}=\ell_{s}^{3} g_{s}$, the two $\mathrm{T}$-dualities may be rewritten in M-theory as,

$$
R_{a} \rightarrow R_{a} v \quad, \quad \ell_{P}^{3} \rightarrow \ell_{P}^{3} v
$$

where $a=i, j, 11$ and $v=l_{p}^{3} / R_{i} R_{j} R_{11}$. As we are free to choose upon which circle we compactify to reach the IIA string theory, this transformation has the general form

$$
R_{i} \rightarrow \frac{\ell_{P}^{3}}{R_{j} R_{k}}, R_{j} \rightarrow \frac{\ell_{P}^{3}}{R_{k} R_{i}}, R_{k} \rightarrow \frac{\ell_{P}^{3}}{R_{i} R_{j}}, \ell_{P}^{3} \rightarrow \frac{\ell_{P}^{6}}{R_{i} R_{j} R_{k}}
$$

where $i, j, k \in\{1 \ldots d+1\}$. In [2] this duality was interpreted in the context of the SYM of Matrix theory as a generalization of the $d=3 \mathrm{~S}$ duality. In fact, it is easy to check that (2.2) is precisely the transformation $T_{i j k} S_{I I B} T_{i j k}$, where $T_{i j k}$ denotes a T-duality on the circles $i, j, k$.

The Weyl group of $E_{d}$ is now generated by reflections corresponding to this duality transformation plus reflections corresponding to permutation of the labelling of the circles $x_{1}, \ldots x_{d}$. To extend the Weyl group from that of $E_{d}$ to $E_{d+1}$ we simply add the reflection that corresponds to the interchange of $x_{d}$ and $x_{11}$. It will be shown later that this additional transformation has an interpretation in the SYM framework involving an exchange of the rank of the gauge group with other quantum numbers.

Our parameter space for M-theory on $T^{d+1}$ consists of the radii, $R_{1}, \ldots R_{d}$, $R_{11}$ and the Planck length $\ell_{P}$, a total of $d+2$ parameters. On the other hand, the root lattice of $E_{d+1}$ is $d+1$-dimensional meaning that the roots will span a hyperplane of codimension 1 . The direction not acted upon by 
the Weyl group corresponds to the quantity,

$$
I=\frac{\ell_{P}^{9}}{R_{11} \prod_{i=1}^{d} R_{i}} \equiv \frac{\ell_{P}^{9}}{R_{11} V_{R}}
$$

which is an invariant under all of the above transformations.

In order to elucidate the algebraic structure of these transformations let us consider a $(d+2)$-dimensional vector space, $\mathcal{M}$ with with the standard orthonormal basis $\left\{m_{a}, a=0, \ldots, d+1\right\}$ and metric $(-1,1, \ldots, 1)$. The unit vector in each direction corresponds respectively to $\left(\log \ell_{P}^{3}, \log R_{i}, \log R_{11}\right)$. Note that the string length $\log \ell_{s}^{2}=m_{0}-m_{d+1}$ is null with respect to this metric.

The Weyl group is generated by the transformations,

$$
\begin{aligned}
& R_{i} \leftrightarrow R_{i+1} \quad \text { for } i=1, \ldots, d-1 \\
& R_{d} \leftrightarrow R_{11} \\
& R_{\alpha} \rightarrow R_{\alpha} v \quad \text { for } \alpha=0,1,2,3
\end{aligned}
$$

where $R_{0}=\ell_{P}^{3}$ and $v=\ell_{P}^{3} / R_{1} R_{2} R_{3}$. In $\mathcal{M}$ these transformations are reflections in the vectors

$$
\begin{aligned}
& \alpha_{0}=m_{0}-m_{1}-m_{2}-m_{3} \\
& \alpha_{i}=m_{i}-m_{i+1} \quad \text { for } i=1, \ldots, d
\end{aligned}
$$

acting on a vector $m \in \mathcal{M}$ in the standard way as

$$
m \rightarrow m-2 \frac{\left(m \cdot \alpha_{a}\right)}{\alpha_{a}^{2}} \alpha_{a}=m-\left(m . \alpha_{a}\right) \alpha_{a} .
$$

Using the above metric it is easy to see that these vectors are the simple roots of $E_{d+1}$ and thus the reflections generate the Weyl group of $E_{d+1}$.

The invariant $I$ is represented in $\mathcal{M}$ as the vector $\mathcal{I}=3 m_{0}-\sum_{1}^{d+1} m_{i}$. $\mathcal{I}$ is orthogonal to all of the roots as it should be, and it has norm $(d-8)$. $\mathcal{I}$ is of some interest to us in our construction as the momentum of a BPS state, when represented by a vector in $\mathcal{M}$, will not generically be in the subspace spanned by the root lattice but will contain a component in the orthogonal $\mathcal{I}$ direction. We thus need to project the vector onto the root lattice subspace. For a vector $m$ this projection is

$$
m \rightarrow m-\frac{(m \cdot \mathcal{I})}{\mathcal{I}^{2}} \mathcal{I}
$$


In terms of the momenta of the BPS states, this projection corresponds to a multiplication by a power of the invariant $I$, and as this power is fixed within a given multiplet the algebraic structure is not modified by this additional factor.

For $d=8, \mathcal{I}$ is null and turns out to be a linear combination of the roots of $E_{9}$. Thus there is no need for a projection onto the hyperplane orthogonal to $\mathcal{I}$, which is just as well as our projection formula is singular precisely in $d=8$.

In this framework we find that all the states discussed in [2] (i.e. the momentum and flux multiplets of $E_{d}$ ) are in the same $E_{d+1}$ multiplet and may all be generated by the above reflections from the BPS state corresponding to KK-momentum in the $x_{11}$ direction, with momentum $1 / R_{11}$. For instance the reflection $\alpha_{d}$ takes this state to the state with momentum $1 / R_{d}$ (the first state in the flux multiplet of [2]). $\alpha_{0}$ then takes this state to $R_{i} R_{j} / \ell_{P}^{3}$ and another reflection in $\alpha_{d}$ takes us to $R_{i} R_{11} / \ell_{P}^{3}$ (the first state in the momentum multiplet).

In $\mathcal{M}$, this fundamental state is represented by the vector $-m_{d+1}$ and after projection onto the hyperplane spanned by the roots we find (for $d \neq 8$ ) the weight vector

$$
\lambda_{d}=-m_{d+1}-\frac{\mathcal{I}}{(d-8)} .
$$

In fact, for all $d, \lambda_{d}$ is the fundamental weight of $E_{d+1}$ dual to the root $\alpha_{d}$. In particular, when $d=9$ (i.e. for $E_{10}$ ) it turns out that $\lambda_{d}$ is minus the null root of the $E_{9}$ subalgebra of $E_{10}$. All of this will be described in more detail in section 3 in terms of T-dual variables more closely related to the SYM matrix model picture to which we now turn.

\subsection{The T-Dual SYM-Like Picture}

To pass from M/IIA-theory to the SYM-like variables of the dual m(atrix) theory, one first performs a T-duality on all the circles of the $d$-torus $T^{d}$ to arrive at what we will refer to as the $\widetilde{\text { II }}$ theory. The parameters in this theory are the dual string coupling constant $\widetilde{g_{s}}$ and the (original and dual) string length $\ell_{s}$. As the matrix theory limit is a 'double scaling limit' of $\widetilde{g}_{s}$ and $\ell_{s}$ (see section 2.3), we will find it convenient to express everything in terms of the lengths $s_{i}$ of the dual $d$-torus, the coupling constant $g^{2}$ of the underlying low-energy SYM theory, and the string length $\ell_{s}^{2}$ of the original IIA theory. $\ell_{s}$ serves to keep track of the $R_{11}=\ell_{s} g_{s}$ of M-theory. Various 
aspects of the matrix theory limit of the $\widetilde{\text { II }}$ U-duality group will be discussed in section 4.

The basic dictionary relating the M-theory variables to those of the $\widetilde{I I}$ theory is

$$
\begin{aligned}
s_{i} & =\frac{\ell_{s}^{2}}{R_{i}} \\
g^{2} & =\widetilde{g}_{s} \ell_{s}^{d-3}=\frac{g_{s} V_{s}}{\ell_{s}^{3}},
\end{aligned}
$$

where $\widetilde{g_{s}}$ is the T-dual string coupling constant

$$
\widetilde{g_{s}}=\frac{g_{s} \ell_{s}^{d}}{V_{R}}=\frac{g_{s} V_{s}}{\ell_{s}^{d}}
$$

and $V_{s}=\prod_{i=1}^{d} s_{i}$ is the volume of the dual torus. The Yang-Mills coupling constant $g^{2}$ follows from matching the low-energy effective action on the Dd-brane,

$$
\begin{aligned}
S & =\frac{1}{\widetilde{g_{s}} \ell_{s}^{d+1}} \int\left(\alpha^{\prime}\right)^{2} F^{2}+\ldots \\
& =\frac{1}{\widetilde{g_{s}} \ell_{s}^{d-3}} \int F^{2}+\ldots,
\end{aligned}
$$

with the standard SYM action $\sim 1 / g^{2}$. In terms of these variables, the invariant (2.3) becomes

$$
I=\frac{g^{4}}{V_{s} \ell_{s}^{2(d-7)}}
$$

Note that this differs from the invariant considered in [2],

$$
I_{E G K R}=\frac{V_{s}^{d-5}}{g^{2(d-3)}},
$$

essentially by its dependence on $R_{11}$, chosen in such a way as to make $I E_{d+1^{-}}$ and not only $E_{d}$-invariant. It turns out (see section 4.5 ) that $I$ is precisely the U-duality invariant that plays an important role in the discussion of black-hole entropies in four and five dimensions.

In order to describe the U-duality symmetries on the II side, let us introduce the quantities $\gamma_{S}$ and $\gamma_{N}$ defined by

$$
\begin{aligned}
\gamma_{S} & =\frac{g^{2}}{\prod_{i=4}^{d} s_{i}} \equiv \frac{g^{2}}{W} \\
\gamma_{N} & =\frac{R_{11}}{R_{d}}=\frac{g^{2} \ell_{s}^{2} s_{d}}{V_{s}} .
\end{aligned}
$$


$\gamma_{S}$ is the effective coupling constant in $d$ dimensions (extrapolated form $d=3$ ), denoted by $g_{\text {eff }}^{2}$ in [2], and sets the scale for the S-duality transformation. $\gamma_{N}$, on the other hand, is the effective $d$-dimensional coupling constant extrapolated from $d=1$ and sets the scale for the N-duality transformation $R_{d} \leftrightarrow R_{11}$ in the $\widetilde{\text { II }}$ picture.

On the $\widetilde{I I}$ side, the Weyl group of the U-duality group is generated by the following three types of transformations (the first two of which have been considered in [2]):

Permutations This is what remains of the geometric $S L(d, \mathbb{Z})$-duality group on a rectangular torus. It acts as

$$
\begin{aligned}
g^{2} & \rightarrow g^{2} \\
s_{i} & \leftrightarrow s_{i+1} \\
s_{j} & \rightarrow s_{j} \quad \text { for } j \neq i, i+1 \\
\ell_{s}^{2} & \rightarrow \ell_{s}^{2} .
\end{aligned}
$$

S-duality This is the $(d+1)$-dimensional generalization of the familiar Sduality transformation of $(3+1)$-dimensional $N=4$ SYM theory

$$
\begin{aligned}
g^{2} & \rightarrow g^{2} \gamma_{S}^{d-5} \\
s_{i} & \rightarrow s_{i} \quad \text { for } i=1,2,3 \\
s_{i} & \rightarrow s_{i} \gamma_{S} \quad \text { for } i=4, \ldots, d \\
\ell_{s}^{2} & \rightarrow \ell_{s}^{2} \gamma_{S}
\end{aligned}
$$

(equivalently, one can replace the first relation by $g_{\text {eff }}^{2} \rightarrow 1 / g_{\text {eff }}^{2}$ ). (2.16) is most succinctly throught of as the transformation $T_{4 \ldots d} S_{I I B} T_{4 \ldots d}$ of the $\widetilde{\text { II }}$ theory, or as $T_{123} S_{I I B} T_{123}$ in the original IIA theory, where $S_{I I B}$ is the S-duality transformation of type IIB string theory and $T_{a b c \ldots}$ denotes a T-duality transformation on the circles $a, b, c, \ldots$ Alternatively, (2.16) follows from applying the dictionary (2.9) to the transformation (2.2) (with $i, j, k=1,2,3$ ),

N-duality This is the counterpart of the $R_{d} \leftrightarrow R_{11}$ transformation in Mtheory and, as we will show, extends the U-duality group of SYM from $E_{d}$ to $E_{d+1}$. The corresponding action on the SYM-like II variables is somewhat more complicated and explicitly given by

$$
g^{2} \rightarrow g^{2} \gamma_{N}^{d-4}
$$




$$
\begin{aligned}
s_{i} & \rightarrow s_{i} \gamma_{N} \quad \text { for } i \neq d \\
s_{d} & \rightarrow s_{d} \\
\ell_{s}^{2} & \rightarrow \ell_{s}^{2} \gamma_{N}
\end{aligned}
$$

and the action on the $\widetilde{I I}$ string couping constant $\widetilde{g_{s}}$ is

$$
\widetilde{g_{s}} \rightarrow \widetilde{g_{s}} \gamma_{N}^{\frac{d-5}{2}}
$$

It is readily checked explicitly that $I$ (2.12) is invariant under all of the above transformations. It can also be checked that this $\mathrm{N}$-duality transformation relates the states in the momentum and flux multiplets to each other (cf. section 2.4), as it should from the M-theory point of view. We need to carefully distinguish between the manner in which the U-duality of Matrix theory on $T^{d}$ arises here and in recent works on the original matrix theory limit of [3]. In our case, the transformations really arise in the $\widetilde{\mathrm{II}}$ theory as an action on the entire collection of $U(N)$ SYM-theories (for all $N$ ) in $d+1$ dimensions, and not for instance on the spectrum of a fixed $U(N)$ SYM theory in $(d+1)+1$ dimensions as in [18, 19]. This is a consequence of working in the DLCQ of M-theory.

In general, there are two ways of looking at the above transformations, as passive transformations, in which one interprets them as acting on BPSstates, or the lattice of quantum numbers, and as active transformations on the parameters $\left(g_{s}, \ell_{s}, s_{i}\right)$ of the string theory. Passively, S-duality exchanges for example the electric and magnetic quantum numbers $\left(e_{i}, m_{j k}\right)$ in the directions $1,2,3$,

$$
\text { Passive S-duality: } e_{1} \leftrightarrow m_{23} \text { etc. }
$$

Thought of actively, S-duality acts, as mentioned above, as

$$
\text { Active S-duality: } T^{d-3} S_{I I B} T^{d-3} \text {. }
$$

The most interesting aspect of N-duality is that, as we will explain in detail in section 4 , in the passive interpretation it acts non-trivially on the rank $N$ of the gauge group $U(N)$ of the underlying low-energy SYM theory, exchanging it with the electric flux quantum number $e_{d}$ in the $d$-direction,

$$
\text { Passive N-duality: } e_{d} \leftrightarrow N \text { etc. }
$$


Thus, N-duality is reminiscent of Nahm-duality [8]. This symmetry has previously been discussed in the context of the U-duality symmetry of $(3+1)$ dimensional SYM theory in [9]. Interpreted actively, on the other hand, $\mathrm{N}$-duality acts as

$$
\text { Active N-duality I: } T^{d-1} S_{I I B} T^{d-1}
$$

where $T^{d-1}$ is shorthand for the $(d-1)$-fold T-duality on the circles transverse to $s_{d}$. This can be seen either from the M-theory origin of the IIB Sduality $S_{I I B}$, or directly in terms of the action on the parameters $\left(s_{i}, s_{d}, \widetilde{g}_{s}, \ell_{s}^{2}\right)$ :

$$
\begin{aligned}
\left(s_{i}, s_{d}, \widetilde{g}_{s}, \ell_{s}^{2}\right) & \stackrel{T^{d-1}}{\longrightarrow}\left(\frac{\ell_{s}^{2}}{s_{i}}, s_{d},{\widetilde{g_{s}}}_{\left.\frac{\ell_{s}^{d-1} s_{d}}{V_{s}}, \ell_{s}^{2}\right)}\right. \\
& \stackrel{S_{I I B}}{\longrightarrow}\left(\frac{\ell_{s}^{2}}{s_{i}}, s_{d}, \frac{V_{s}}{{\widetilde{g_{s}}}_{\ell_{s}}^{d-1} s_{d}}, \frac{\ell_{s}^{d+1} \widetilde{g_{s}} s_{d}}{V_{s}}\right) \\
& \stackrel{T^{d-1}}{\longrightarrow}\left(s_{i} \gamma_{N}, s_{d},{\widetilde{g_{s}}}^{\frac{d-5}{2}}, \ell_{s}^{2} \gamma_{N}\right)
\end{aligned}
$$

In particular, in the matrix theory limit (cf. section 2.3), the N-dual theory becomes effectively $(1+1)$-dimensional,

$$
\text { Active N-duality II: } \mathrm{SYM}_{d+1} \rightarrow \mathrm{SYM}_{1+1} \text {. }
$$

precisely as in 13]. We will say more about these active and passive interpretations of N-duality in section 4.

What is probably not at all obvious at this point is that this N-duality really extends the U-duality group from $E_{d}$ to $E_{d+1}$. This is most easily verified within the appropriate generalization of the algebraic framework introduced in [2] which we will construct in section 3.

\subsection{The Matrix Theory Limit and the DLCQ of M-Theory}

Shortly after the original BFSS m(atrix) theory proposal [3] for a nonperturbative definition of M-theory, it was realized that toroidally compactified string theory is related to (the large $N$ limit of) maximally supersymmetric $U(N)$ Yang-Mills theory on the dual torus [20, 3] (this prescription has recently been reanalyzed in [11], with some rather striking consequences). In fact, this picture follows naturally from T-duality, mapping a configuration of $N$ D0-branes on the IIA side to that of $N \mathrm{D} d$-branes on the dual II (= IIA or IIB) side. In this setting, the SYM theory arises as the low-energy effective action on the world-volume of the $\mathrm{D} d$-branes. 
For several reasons, however, this picture is incomplete and not completely satisfactory.

First of all, for $d>3$ the SYM theory is not renormalizable and thus gives an incomplete description of the physics at short distances. This (among other things) led to the search for more general (field, string, ...? ) theories on the world-volumes of extended objects in string theory or M-theory which are decoupled from the ten- or eleven-dimensional bulk dynamics. Subsequently, a matrix theory description for $d=4,5$ was proposed in terms of a $(2,0)$ supersymmetric field theory on the M5-brane [18, 17] and a theory of 'microstrings' localized on the world-volume of the NS5-brane [21, 17, 13] respectively.

Secondly, many of the attractive features of the original BFSS theory (and its descendants) appear strictly speaking only in the infinite momentum frame $N \rightarrow \infty$ limit which is awkward to deal with directly. Therefore the suggestion by Susskind [] that the finite $N$ matrix model might actually give a complete description of the discrete light cone quantization (DLCQ) of M-theory in the sector carrying $N$ units of light-like momentum, was particularly attractive.

Very recently, Sen and Seiberg [5, 6] combined these two issues to provide a) substantial evidence for Susskind's conjecture and b) a systematic way of deriving the matrix models proposed for $d \leq 5$. In particular, therefore, this means that one can consider the 'double scaling' matrix theory limit $\ell_{s} \rightarrow 0, g_{s} \rightarrow 0$ of M-theory compactified on space-like circles (a setting in which the $E_{d+1} \mathrm{U}$-duality is manifest on the M-theory side) and reach the finite $N$ DLCQ of M-theory in the limit, assigning a physically meaningful interpretation to the finite $N$ versions of the matrix models proposed for $d \leq 5$. One might perhaps have expected the U-duality group of a light-like torus to differ from that of a space-like torus. However, this is apparently not the case [15, 22].

In terms of the M/IIA-theory variables, this matrix theory limit is the limit $\ell_{s} \rightarrow 0, g_{s} \rightarrow 0$, keeping fixed the radii $R_{i}$ measured in Planck units $\ell_{P}$, and the ratio $\ell_{s}^{3} / g_{s}$. In particular, therefore, the ratios $R_{i} / \ell_{s}^{2}$ are fixed (thus $R_{i} \rightarrow 0$ ) and the matrix theory limit is actually

$$
\begin{array}{ll}
\text { IIA Matrix Theory Limit: } & \ell_{s} \rightarrow 0, g_{s} \rightarrow 0 \\
& R_{i} / \ell_{P}=\text { constant } \\
& R_{i} / \ell_{s}^{2}=\text { constant } .
\end{array}
$$

The more transparent interpretation of this on the $\widetilde{\text { II }}$ side is that one is 
taking the same limit, keeping fixed the parameters (2.9), i.e.

$$
\begin{array}{ll}
\text { II Matrix Theory Limit: } \quad \ell_{s} \rightarrow 0, g_{s} \rightarrow 0 \\
s_{i}=\text { constant } \\
g^{2}=\text { constant } .
\end{array}
$$

This limit captures correctly the SYM degrees of freedom and is also natural from the D-brane probe point of view 23 .

From (2.9) we can read off that in $d \leq 2$ the matrix theory limit amounts to letting $\ell_{s} \rightarrow 0$ and $\widetilde{g_{s}} \rightarrow 0$, while in $d=3$ one has $\ell_{s} \rightarrow 0$ with $\widetilde{g_{s}}$ constant. In both cases, this amounts to decoupling of the dynamics on the branes from the bulk dynamics (in particular, gravity). In these cases, thus, the SYM picture provides an adequate description of the DLCQ of M-theory.

In $d=4, \widetilde{g_{s}} \rightarrow \infty$ in the matrix theory limit, and thus one can certainly not ignore the coupling to the bulk fields. However, as $\widetilde{g}_{s} \ell_{s}$ is constant in the matrix theory limit, this suggests the emergence of a new 'eleventh' direction in the $\widetilde{\mathrm{II}}$ theory with radius $\widetilde{R}_{11}=\ell_{s} \widetilde{g}_{s}$, whose $\widetilde{\mathrm{D} 0}$ branes are the instantons on the D4-brane worldvolume [18]. The dynamics on the fivebrane (the $(2,0)$ supersymmetric theory) of this $\widetilde{M}$-theory also decouples from the bulk dynamics because ${\widetilde{\ell_{P}}}^{3}=\ell_{s}{\widetilde{g_{s}}}^{3} \rightarrow 0$ [17].

Also in $d=5, \widetilde{g_{s}}$ diverges in the matrix theory limit. In this case, one can perform an S-duality to convert the $N$ D5-branes to $N$ solitonic NS5-branes. The new string coupling constant $\widehat{g_{s}}=1 / \widetilde{g_{s}} \rightarrow 0$, while the new string length $\hat{\ell}_{s}^{2}=g^{2}$ remains constant, suggesting that at these energies the theory is described by string-excitations confined to the NS5-brane worldvolume [21, 17, 13.

In $d>5$, in spite of a number of attempts [24], no satisfactory matrix theory formulation is known [6, 5]. However, one expects that, if found, this theory will reduce at low energies to SYM theory in $(d+1)$-dimensions, and that (part of) the BPS spectrum can be reliably determined at low energies. With this in mind, we will make use of the matrix theory limit in section 4 to extract information about the U-duality groups of matrix theories from those of the $\widetilde{I I}$ theories.

\subsection{Dd-Brane Backgrounds and Bound States}

As we will frequently make use of the BPS mass formulae in the matrix theory limit in subsequent sections, we briefly recall the relevant equations. 
Let us consider $N$ D0-branes on the M/IIA-side. Upon passing to the $\widetilde{\mathrm{II}}$ side, this gives a configuration of $N \mathrm{D} d$-branes wrapping the (dual) torus $T^{d}$. This state has a mass

$$
M_{N d}=\frac{N}{R_{11}}=\frac{N V_{s}}{\widetilde{g_{s}} \ell_{s}^{d+1}} .
$$

In order to analyze the energy of BPS bound states in the matrix theory (SYM) limit, it will be convenient to reexpress (2.27) in terms of the $\widetilde{\mathrm{II}}$ variables $\left(g^{2}, s_{i}, \ell_{s}^{2}\right)$ as

$$
M_{N d}=\frac{N V_{s}}{g^{2} \ell_{s}^{4}}
$$

In the matrix theory limit, this state becomes infinitely heavy and should thus be treated as a background configuration whose energy is to be subtracted in the calculation of bound state energies.

BPS bound states of the background Dd-branes with other objects in the theory (KK modes, wound strings, D-branes, solitons) can basically be of two kinds. There are bound states which preserve half of the original supersymmetries of the IIA theory, i.e. no further supersymmetries are broken beyond those broken by the background. These are non-threshold bound states with non-zero binding energy and, by the saturation of the Bogomoln'y bound for the BPS state, the energy $E$ of the bound state is related to the background mass (2.28) and the mass $M$ of the other object by

$$
E^{2}=M_{N d}^{2}+M^{2}
$$

Examples of such bound states are D0-KK and D0-D2 systems on the IIA side, and thus bound states of the background $\mathrm{D} d$-branes with either wound NS strings or wrapped $\mathrm{D}(d-2)$-branes on the II side. An NS string wound $p$ times around the circle $s_{i}$ has a mass $M=p s_{i} / \ell_{s}^{2}$, and thus the bound state energy is

$$
E^{2}=\frac{N^{2} V_{s}^{2}}{g^{4} \ell_{s}^{8}}+\frac{p^{2} s_{i}^{2}}{\ell_{s}^{4}}
$$

Calculating the energy in the matrix theory limit $\ell_{s} \rightarrow 0$ and subtracting the background one finds

$$
\begin{aligned}
E_{S Y M} & =\lim _{\ell_{s} \rightarrow 0}\left(\sqrt{\frac{N^{2} V_{s}^{2}}{g^{4} \ell_{s}^{8}}+\frac{p^{2} s_{i}^{2}}{\ell_{s}^{4}}}-\frac{N V_{s}}{g^{2} \ell_{s}^{4}}\right) \\
& =\frac{p^{2} g^{2} s_{i}^{2}}{2 N V_{s}}
\end{aligned}
$$


i.e. precisely the energy of an electrix flux state of SYM theory. More generally, whenever $M \sim \ell_{s}^{-2}+O\left(\ell_{s}^{-1}\right)$ as $\ell_{s} \rightarrow 0$, one will obtain a finite (and non-zero) energy

$$
E_{S Y M}=\frac{M^{2}}{2 M_{N d}}
$$

in the matrix theory limit (we will omit this factor of $1 / 2$ in the following). In particular this is also the case for the p-wrapped $\mathrm{D}(d-2)$-brane with mass $(i, j$ are the unwrapped directions)

$$
M=\frac{p V_{s}}{s_{i} s_{j} \widetilde{g_{s}} \ell_{s}^{d-1}}=\frac{p V_{s}}{s_{i} s_{j} g^{2} \ell_{s}^{2}},
$$

leading to the standard magnetic flux bound state energy

$$
E_{S Y M}=\frac{p^{2} V_{s}}{N g^{2} s_{i}^{2} s_{j}^{2}}
$$

in the limit.

The other class of bound states of interest to us are so-called threshold bound states preserving just one quarter of the original supersymmetries. These are subtle objects in general, but the prototypical examples here are D0NS winding and D0-D4 states on the IIA side, corresponding to momentum states $M=p / s_{i}$ and $\mathrm{D} d-\mathrm{D}(d-4)$ bound states on the II side. As the binding energy is zero, these satisfy the linear bound state energy relation

$$
E=M_{N d}+M
$$

so that

$$
E_{S Y M}=\lim _{\ell_{s} \rightarrow 0} M .
$$

This will be finite and non-zero in the limit if $M \sim$ constant $+O\left(\ell_{s}\right)$ as $\ell_{s} \rightarrow 0$. This is the case, in particular, for the above momentum state, but also for the $p$-wrapped $\mathrm{D}(d-4)$-brane which has mass

$$
M=\frac{p V_{s}}{\widetilde{g}_{s} \ell_{s}^{d-3} s_{i_{1}} s_{i_{2}} s_{i_{3}} s_{i_{4}}}=\frac{p V_{s}}{g^{2} s_{i_{1}} s_{i_{2}} s_{i_{3}} s_{i_{4}}}
$$

and represents an instanton in the matrix theory. We will encouter more exotic examples of matrix theory bound states in section 4 .

It is now also easy to see in which sense N-duality connects the flux and momentum multiplets of [2]. E.g. starting with the momentum state with 
$M=1 / s_{i}$ one obtains the magnetic flux state with mass $M=V_{s} / g^{2} \ell_{s}^{2} s_{i} s_{d}$. However, as N-duality acts non-trivially on the background, in order to have a SYM-like interpretation in both cases, one needs to start with a configuration with non-zero momentum in both the 11- and the $d$-direction. This will also be discussed in more detail in section 4, though already now it is clear that all the one-particle states in the flux and momentum multiplets can be generated from the background (vacuum) state $\mathcal{N}=1 / R_{11}$ (as noted in section 2.1) or

$$
\mathcal{N}=\frac{V_{s}}{g^{2} \ell_{s}^{4}}
$$

by U-dualities. In fact (cf. section 3.4 ), $\mathcal{N}$ is precisely the highest weight state in the orbit of the U-duality group, and that the highest weight state corresponds to the vacuum does probably not come as a surprise.

At various points in this paper we talk about BPS field configurations in terms of their (gravitational) masses. For $d \leq 6$ this is perfectly legitimate but for $d>6$ the concept of mass in the $(9-d)+1$ dimensional noncompact spacetime is ill-defined due to the lack of asymptotic flatness of the configurations. Nevertheless, also for $d>6 E_{S Y M}$ makes sense and is the appropriate quantity to consider for the purposes of (formally) discussing the action of U-duality on the BPS states.

\section{Extending the U-Duality Group from $E_{d}$ to $E_{d+1}$ : The Algebra}

\subsection{The Algebraic Set-UP}

In [2], a convenient framework for discussing various algebraic aspects of U-duality was introduced. We will make use of this framework here, with some modifications which make the construction more natural from an Mtheory $/ E_{d+1}$ point of view.

Thus consider a $(d+2)$-dimensional vector space $\mathcal{Y}$ spanned by $\left(\log g^{2}, \log s_{i}, \log \ell_{s}^{2}\right)$ and introduce the $\widetilde{\text { II }}$ counterparts $\left\{y_{a}\right\}$ of the M-theory vectors $\left\{m_{a}\right\}=$ $\left\{\log \ell_{P}^{3}, \log R_{i}, \log R_{11}\right\}$, namely

$$
\begin{aligned}
y_{0} & =\log g^{2}+3 \log \ell_{s}^{2}-\log V_{s} \\
y_{i} & =\log \ell_{s}^{2}-\log s_{i} \\
y_{d+1} & =\log g^{2}+2 \log \ell_{s}^{2}-\log V_{s} \equiv-\log \mathcal{N} .
\end{aligned}
$$


One can now postulate (or deduce from the flat M-theory metric) the metric

$$
y_{a} \cdot y_{b}=\eta_{a b} \quad \eta_{a b}=\operatorname{diag}(-1,+1, \ldots,+1) .
$$

In terms of the natural $\widetilde{\mathrm{II}}$ or matrix theory variables $\left(\widetilde{g_{s}}, s_{i}, \ell_{s}^{2}\right)$ or $\left(g^{2}, s_{i}, \ell_{s}^{2}\right)$ this becomes the off-diagonal metric $G_{a b}$ with non-zero components

$$
\begin{array}{rccl}
G_{\widetilde{g_{s}} \widetilde{g_{s}}}=2 & \text { or } & G_{g^{2} g^{2}}=(5-d) \\
G_{\widetilde{g}_{s} \ell_{s}^{2}}=-1 & \text { or } \quad & G_{g^{2} \ell_{s}^{2}}=-1 \\
& G_{s_{i} s_{i}}=1 & .
\end{array}
$$

In $\mathcal{Y}$, the invariant $I(2.12)$ is represented by the vector

$$
\mathcal{I}=2 \log g^{2}-\log V_{s}-(d-7) \log \ell_{s}^{2},
$$

with norm

$$
\mathcal{I} \cdot \mathcal{I}=(d-8)
$$

We thus see that for $d \leq 7$ the induced metric on the orthogonal complement to $\mathcal{I}$ in $\mathcal{Y}$ is positive definite, while for $d=9$ it is indefinite. The case $d=8$ is special and will lead to a degenerate metric on root space. All this is, of course, consistent with the structure one expects for the root space of $E_{d+1}$.

\subsection{The Weyl Group of $E_{d+1}$}

It is now possible to realize the transformations (2.15 2.17) as reflections in the vector space $\mathcal{Y}$. Indeed, these transformations are reflections along the vectors

$$
\begin{aligned}
\alpha_{0} & =\log g^{2}-\log W=\log \gamma_{S} \\
\alpha_{i} & =\log s_{i+1}-\log s_{i} \quad \text { for } i=1, \ldots, d-1 \\
\alpha_{d} & =-\log \gamma_{N}
\end{aligned}
$$

respectively. In this setting, the invariance of $I$ is expressed by

$$
\alpha_{0} \cdot \mathcal{I}=\alpha_{i} \cdot \mathcal{I}=\alpha_{d} \cdot \mathcal{I}=0
$$

The following properties of the $\alpha_{a}$ are now readily verified:

$$
\begin{aligned}
\left(\alpha_{a}\right)^{2} & =2 \quad \forall a=0, \ldots, d \\
\alpha_{i} \cdot \alpha_{i+1} & =-1 \quad \forall i=1, \ldots, d-1 \\
\alpha_{0} \cdot \alpha_{3} & =-1 .
\end{aligned}
$$


Thus the $\left\{\alpha_{a}\right\}$ represent precisely the root system and Dynkin diagram of $E_{d+1}$ and the U-duality symmetries (2.15-2.17) generate the Weyl group of $E_{d+1}$.

Let us be slightly more explicit about this for the 'exotic' cases $E_{9}$ and $E_{10}$. $E_{9}=\widehat{E_{8}}$ is the affine algebra of $E_{8}$. Its root system is usually presented in terms of the vectors

$$
\begin{aligned}
\bar{\alpha}_{i} & =\left(\beta_{i}, 0,0\right) \\
\delta & =(0,0,1) \\
\kappa & =(0,1,0)
\end{aligned}
$$

where $\beta_{i}, i=0, \ldots, d-1=7$ are the simple roots of $E_{8}$ and the nonvanishing scalar products are

$$
\bar{\alpha}_{i} \cdot \bar{\alpha}_{j}=\beta_{i} \cdot \beta_{j} \quad \delta . \kappa=1 .
$$

The simple roots are chosen to be $\bar{\alpha}_{i}$ and

$$
\bar{\alpha}_{8}=(-\psi, 0,1),
$$

where $\psi$ is the highest root of $E_{8}$.

To identify this structure in the present context, we can first of all identify the $\bar{\alpha}_{i}$ with our $\alpha_{i}$ for $i=0, \ldots, 7$. Explicit calculation shows that the remaining root $\alpha_{d=8}$ (3.6) can be written as

$$
\alpha_{d=8}=-\psi+\mathcal{I}
$$

In particular, therefore, $\mathcal{I}$ is a linear combination of the roots,

$$
\mathcal{I}=3 \alpha_{0}+2 \alpha_{1}+4 \alpha_{2}+6 \alpha_{3}+5 \alpha_{4}+4 \alpha_{5}+3 \alpha_{6}+2 \alpha_{7}+\alpha_{8} .
$$

so that we can identify $\mathcal{I}$ with a multiple of $\delta$,

$$
\mathcal{I}=p \delta
$$

Thus we have $\bar{\alpha}_{8}=-\psi+\mathcal{I} / p$ and $\alpha_{d=8}=-\psi+p \delta$. Finally, there is another null-direction in $\mathcal{Y}$, orthogonal to all the roots of $E_{8}$, spanned by

$$
\mathcal{K}=\mathcal{I}-2 \log \mathcal{N} .
$$

$\mathcal{I}$ and $\mathcal{K}$ have a non-zero scalar product, $\mathcal{I} \cdot \mathcal{K}=-2$, and we we can thus identify $\mathcal{K}=q \kappa$. The scalar product $\kappa . \delta=1$ now implies that $p q=-2$ or that

$$
\mathcal{K}=-\frac{2}{p} \kappa
$$


At this point, $p$ is undetermined. However, we will see below that both the considerations regarding $E_{10}$ and those regarding the representation of the U-duality group in $d=8$ imply that $p=1$ so that

$$
\begin{aligned}
\alpha_{d=8} & =\bar{\alpha}_{8}=(-\psi, 0,1) \\
\mathcal{I} & =\delta \\
\mathcal{K} & =-2 \kappa
\end{aligned}
$$

Let us now turn to the U-duality group $E_{10}$ that we obtain for $d=9$. $E_{10}$ is a hyperbolic Lie algebra (meaning a Kac-Moody algebra which is such that upon removal of any node from its Dynkin diagram one obtains either finitedimensional or affine Kac-Moody algebras - for a digestible introduction to $E_{10}$ see [25]). Recall that we have the roots $\left\{\alpha_{a}, a=0, \ldots, 9\right\}$, of which we identify the $\alpha_{i}, i=0, \ldots, 7$, with the simple roots of $E_{8}$. It is conventional to replace $\alpha_{8}$ by the null root $\delta$ of $E_{9}$, i.e. by $\mathcal{I}_{d=8} / p$, where $\mathcal{I}_{d=8}$ is the eightdimensional invariant, given in terms of roots in (3.13). Its scalar product with the hyperbolic root $\alpha_{d=9}$ is known to be

$$
\alpha_{d=9} \cdot \delta=-1
$$

but, as $\mathcal{I}_{d=8}=\alpha_{8}+\ldots$, one has $\alpha_{d=9} \cdot \mathcal{I}_{d=8}=-1$, and thus we find $p=1$. The other null direction orthogonal to $\mathcal{I}_{d=9}$ and the roots of $E_{8}$ is spanned by $\alpha_{d=9}+\delta$. This is directly related to the $\kappa$ of $E_{9}$. In fact, while $\mathcal{K}_{d=8}$ (3.15) constituted an independent direction in $E_{9}$, in $E_{10}$ it can be expressed in terms of the roots as

$$
\mathcal{K}_{d=8}=2\left(\alpha_{d=9}+\delta\right)
$$

so that

$$
\kappa=-\left(\alpha_{d=9}+\delta\right)
$$

with

$$
\kappa^{2}=0, \quad \kappa . \delta=1
$$

\subsection{Fundamental Weights of $E_{d+1}$}

In [2], primarily two kinds of BPS states were investigated, the momentum multiplet, associated on the M-theory side with longitudinally wrapped branes, and the flux multiplet, corresponding to KK modes (electric flux) or transversally wrapped branes (magnetic flux). These were shown to transform seperately under the $E_{d} \subset E_{d+1}$ duality group (or rather, its Weyl group) generated by S-duality and permutations. 
From the M-theory point of view, it is of course obvious that including $R_{11} \leftrightarrow R_{d}$ permutations (upon which the distinction between longitudinal and transverse branes disappears) will connect these two multiplets, and we have also already indicated this explicitly in sections 2.1 and 2.4 above. It follows that the two $E_{d}$-multiplets are part of one $E_{d+1}$ multiplet, and in the following we will identify the corresponding highest weight states, deferring to section 4 a discussion of the new BPS states one obtains in that way (states that are neither in the momentum nor in the flux multiplet of $\left.E_{d} \subset E_{d+1}\right)$.

As all the $E_{d+1}$ are simply laced, the fundamental weights $\left\{\lambda_{a}\right\}$ dual to the simple roots $\left\{\alpha_{b}\right\}$ (3.6) of $E_{d+1}$ are characterized by $\lambda_{a} \cdot \alpha_{b}=\delta_{a b}$. Furthermore, looking for solutions to these equations within the $(d+2)$-dimensional vector space $\mathcal{Y}$, for $d \neq 8$ we need to require that the $\lambda_{a}$ be orthogonal to the invariant $\mathcal{I}_{d}$. For $d=8$, on the other hand, the fundamental weights are only determined up to addition of a multiple of the null root $\delta$ (or the invariant $\mathcal{I}_{d=8}$ ). As the null root is orthogonal to all the roots of $E_{9}$, this does not change the corresponding highest weight representation. For reference purposes, we here provide explicit expressions for all the fundamental weights $\left\{\lambda_{a}\right\}$.

For $d \neq 8$, the fundamental weights dual to the simple roots (3.6) and orthogonal to $\mathcal{I}_{d}$ in $\mathcal{Y}$ are

$$
\begin{aligned}
\lambda_{0}= & \frac{1}{(d-8)}\left[(2-d) \log g^{2}+(d-5) \log V_{s}+3 \log \ell_{s}^{2}\right] \\
\lambda_{i}= & \frac{i}{2(d-8)}\left[(4-d) \log g^{2}+(d-6) \log V_{s}+2 \log \ell_{s}^{2}\right]-\log V_{s, i} \quad i=1,2,3 \\
\lambda_{j}= & \frac{1}{(d-8)}\left[(-3 d+3 j+6) \log g^{2}+(3 d-j-15) \log V_{s}+(9-j) \log \ell_{s}^{2}\right] \\
& -\log V_{s, j} \quad j=4, \ldots, d-1 \\
\lambda_{d}= & \frac{1}{(d-8)}\left[(6-d) \log g^{2}+(d-7) \log V_{s}+(9-d) \log \ell_{s}^{2}\right]
\end{aligned}
$$

where $V_{s, i}$ denotes the partial volume $\prod_{a=1}^{i} s_{a}$.

As mentioned above, the fundamental weights are not unique in $d=8$, but a convenient set of representatives is

$$
\begin{aligned}
\lambda_{0} & =-\log g^{2}+\log V_{s}-3 \log \ell_{s}^{2} \\
\lambda_{i} & =i\left(\log V_{s}-\log g^{2}-2 \log \ell_{s}^{2}\right)-\log V_{s, i} \quad i=1,2,3 \\
\lambda_{j} & =-3 \log g^{2}+3 \log V_{s}+(j-9) \log \ell_{s}^{2}-\log V_{s, j} \quad j=4,5,6,7 \\
\lambda_{d=8} & =\log V_{s}-\log g^{2}-2 \log \ell_{s}^{2}=\log \mathcal{N} .
\end{aligned}
$$

In general, because of the necessity to project the weights, and because the invariant $I$ is dimensionful, the correspondence between the masses of BPS 
states and weights of the U-duality group is somewhat indirect. Both of these complications are absent in $d=8$, however, and some conclusions can be drawn directly from looking at the highest weight states. It is easy to see that the mass-dimensions of the highest weight states of the fundamental representations are

$$
\left[\lambda_{0}\right]=3,\left[\lambda_{i}\right]=2 i,\left[\lambda_{j}\right]=9-j,\left[\lambda_{d=8}\right]=1 .
$$

Thus the only highest weight (positive integral linear combination of the fundamental weights) having a mass dimension of an energy is $\lambda_{d=8}$, and this is indeed the highest weight state of the half-BPS configurations. It follows that the U-duality orbits of other BPS bound states are necessarily described in terms of higher powers of the mass. We see that this fact, which is well known for $E_{d \leq 8}$ (see e.g. [26]), follows rather readily for $E_{9}$ within the present framework.

\subsection{The Highest Weights of the U-Duality Group $E_{d+1}$}

The fundamental weight we will primarily be interested in is the weight $\lambda_{d}$ dual to the root $\alpha_{d}$ generating the $\mathrm{N}$-duality transformation (2.17). It is always of the form

$$
\lambda_{d}=\log \mathcal{N}+\mu_{d} \mathcal{I}_{d}
$$

for some $d$-dependent constant $\mu_{d}$, where $\mathcal{N}$ was defined in (2.38), and as such represents the precise SYM counterpart of the M-theory longitudinal momentum state $1 / R_{11}$ discussed in section 2.1. As explained in section $2.4, \mathcal{N}$ just represents the $\mathrm{D} d$-brane background of the $\widetilde{\mathrm{II}}$ theory, i.e. the vacuum, a not unexpected feature of a highest weight state.

Of course, as a result of the enlargement of the geometric duality group on the M-theory side from $S L(d, \mathbb{Z})$ to $S L(d+1, \mathbb{Z})$, we also find states which appear neither in the momentum nor in the flux multiplets of $E_{d}$. In $d \leq 5$ dimensions, only one new state, the highest weight state $\mathcal{N}$, is needed to unify the momentum and flux multiplets. But e.g. for $d=6$, instead of the $27+27=54$ flux and momentum states in the $\overline{\mathbf{2 7}} \oplus \mathbf{2 7}$ of $E_{6}$ [2], we find 56 states in the Weyl group orbit of the $\mathbf{5 6}$ of $E_{7}$. And for $d=7$, instead of the $56+126=182$ flux and momentum states in the $\mathbf{5 6} \oplus \mathbf{1 3 3}$ of $E_{7}$ we find 240 states in the Weyl group orbit of the $\mathbf{2 4 8}$ of $E_{8}$. We will return to this in section 4 .

In $d=8$, the U-duality group is $E_{9}=\widehat{E_{8}}$. Again, the highest weight of 
interest is the fundamental weight dual to $\alpha_{d}$, i.e. $\lambda_{d}=\log \mathcal{N}$ or 3.15

$$
\lambda_{d=8}=\frac{1}{2}(\mathcal{I}-\mathcal{K})
$$

Using (3.14) and (3.16), we can write this as

$$
\lambda_{d=8}=\frac{1}{2}\left(p \delta+\frac{2}{p} \kappa\right)=\left(0, \frac{1}{p}, \frac{p}{2}\right)
$$

We can now fix $p$ (and hence the level of the representation) by an argument analogous to (but more conclusive than) that employed in [2 for $d=9$. First of all, we observe that demanding that the level be an integer imposes the requirement $(1 / p) \in \mathbb{Z}$. Secondly, since

$$
\alpha_{d=8}=-\psi+p \delta
$$

the requirement that the affine algebra have an integer moding imposes $p \in \mathbb{Z}$. These two conditions are uniquely solved by $p=1$ (as we also found via $E_{10}$ in section 3.4). Thus the level is $k=1$, and in fact the representation $\lambda_{d=8}$ is the unique integrable representation of $E_{9}$ at $k=1$. Why one should find a unitarizable representation (or if there is a good reason for this) remains a mystery at this point [2].

Finally, for $d=9$, i.e. $E_{10}$, the highest weight state is

$$
\lambda_{d=9}=2 \log V_{s}-3 \log g^{2} .
$$

As $\lambda_{d=9}$ is null and orthogonal to all the simple roots apart from $\alpha_{d=9}$, it is proportional to the null root $\delta$ of $E_{9}$, and $\alpha_{d} \cdot \lambda_{d}=1$ determines

$$
\lambda_{d=9}=-\delta .
$$

While this is certainly a distinguished highest weight of $E_{10}$, in the absence of some information about this representation of $E_{10}$ (which we have not been able to find) there is nothing that we can add to this at this point.

\section{Interpretation AND ApPlications}

We have seen in the considerations of the previous sections that N-duality, as defined in (2.17), and concretely realized as the transformation $T^{d-1} S_{I I B} T^{d-1}$ (2.22), is the natural transformation to add to the Weyl group of $E_{d}$ to 
extend it to the Weyl group of the full U-duality group $E_{d+1}$. In fact, Nduality is precisely the transformation associated with the $(d+1)$ 'th node of the Dynkin diagram of $E_{d+1}$.

In order to clarify the general structure of the N-duality transformation (2.17), in this section we will describe in more detail how it acts on the BPS quantum numbers, in which sense it can be regarded as a Nahm-like duality, and in which sense it provides a $(1+1)$-dimensional or $(2+1)$-dimensional picture of BPS states (and perhaps not only of those). We also relate the U-duality invariant $I(2.3,2.12)$ to the expression for entropies of black holes in four and five dimensions, we look at the BPS spectra in $d=6$ and $d=7$ and provide concrete interpretations for some of the states we find.

\subsection{U-Duality, Nahm Duality, and the Matrix Theory Limit}

Let us introduce the units of electric, magnetic, and momentum flux $\mathcal{E}_{i}$, $\mathcal{M}_{i j}, \mathcal{P}_{i}$ as well as the quantity $\mathcal{N}$, defined by

$$
\begin{aligned}
\mathcal{N} & =\frac{V_{s}}{g^{2} \ell_{s}^{4}} \\
\mathcal{P}_{i} & =\frac{1}{s_{i}} \\
\mathcal{E}_{i} & =\frac{s_{i}}{\ell_{s}^{2}} \\
\mathcal{M}_{i j} & =\frac{V_{s}}{g^{2} \ell_{s}^{2} s_{i} s_{j}} \quad i<j .
\end{aligned}
$$

These correspond to the $(d+1)$-dimensional KK and wrapped M2-brane masses on the M-theory side. Let us denote the corresponding quantum numbers by $\left(N, p_{i}, e_{i}, m_{i j}\right)$ (in general, we should of course also introduce analogous quantum numbers for wrapped M5-brane states etc.). S-duality in the $i, j, k$ directions exchanges $\mathcal{E}_{i}$ and $\mathcal{M}_{j k}$, or $e_{i}$ and $m_{j k}$, leaving all the other quanta invariant, while N-duality acts as

$$
\begin{array}{cl}
\mathcal{E}_{d} \leftrightarrow \mathcal{N} & \text { i.e. } e_{d} \leftrightarrow N \\
\mathcal{P}_{i} \leftrightarrow \mathcal{M}_{i d} & \text { i.e. } p_{i} \leftrightarrow m_{i d}
\end{array}
$$

for $i<d$, leaving the other quantum numbers invariant. We are not being careful with signs here but these can be readily deduced from the T-duality rules for D-branes given in [27]. For example for $d=3$ one finds that the ten quantum numbers $\left(N, p_{i}, e_{i}, m_{i}\right)$, corresponding to the background 
D3-branes, KK modes, and fundamental and D-string winding numbers, transform as $(a=1,2)$

$$
\begin{aligned}
N \rightarrow-e_{3} & p_{a} \rightarrow \epsilon_{a b} m_{b} \\
e_{3} \rightarrow N & m_{a} \rightarrow \epsilon_{a b} p_{b}
\end{aligned}
$$

with $\left(p_{3}, m_{3}, e_{a}\right)$ invariant, much as in [9]. Arranging the ten quantum numbers as an anti-symmetric $(5 \times 5)$ matrix, one can verify that this transformation is indeed implemented by an $S L(5, \mathbb{Z})$-rotation, as expected.

In particular, we recover the fact mentioned before that $\mathrm{N}$-duality relates states in the momentum multiplet to magnetic flux states in the flux multiplet. However, the most interesting effect of this transformation is encoded in the first line of (4.2). As we have seen, $\mathcal{N}$ is to be interpreted as measuring the bound state energy of the background $\mathrm{D} d$-branes, and therefore the corresponding quantum number is the rank of the gauge group.

A duality action in SYM theory acting non-trivially on the rank of the gauge group is not as unfamiliar as it may at first seem, at least in the context of D-branes. Consider for example a bound state of $N$ D $d$-branes on $T^{d}$ (giving rise to $U(N)$ SYM theory) together with a $\mathrm{D}(d-2)$-brane wound $M$ times around the $3,4, \ldots, d$ directions. Then a T-duality on the circles 1 and 2 will exchange the $\operatorname{rank} N$ with the magnetic quantum number $M$. This is related to the $\mathrm{N}$-duality transformation above (which exchanges the rank and electric quantum numbers) by S-duality.

Concretely, in our case, we can consider the non-threshold electric flux bound state of $N$ background $\mathrm{D} d$-branes with a fundamental NS string $\mathrm{F} 1_{d}$ wound $e_{d}$ times around the $d$ 'th circle. N-duality will map this to

$$
\text { Example I: } \quad N \mathrm{D} d+e_{d} \mathrm{~F} 1_{d} \begin{aligned}
\stackrel{T^{d-1}}{\longrightarrow} & N \mathrm{D} 1_{d}+e_{d} \mathrm{~F} 1_{d} \\
& \stackrel{S_{I I B}}{\longrightarrow} N \mathrm{~F} 1_{d}+e_{d} \mathrm{D} 1_{d} \\
& \stackrel{T^{d-1}}{\longrightarrow} N \mathrm{~F} 1_{d}+e_{d} \mathrm{D} d,
\end{aligned}
$$

and we thus see explicitly that it exchanges the quantum numbers $\left(e_{d}, N\right)$, mapping the corresponding BPS states to each other. This is reminiscent of the Nahm-duality transformation for instantons on $T^{4}$ which provides an isomorphism between the rank $N$ instanton number $k$ and rank $k$ instanton number $N$ instanton moduli spaces [8] (and precisely this Nahm duality arises in the consideration of $\mathrm{D}(p+4)-\mathrm{D} p$ brane systems [28, 29]).

Of course, changing $N$ also amounts to changing the longitudinal momentum sector of M(atrix) theory described by its DLCQ. Thus, N-duality actually 
relates BPS states corresponding to different gauge groups and, as suggested in [7], the theories for different values of $N$ should perhaps be combined into some larger theory. As mentioned in the introduction, N-duality would then be a signature of the Lorentz invariance of this theory. Alternatively, we note that at least in the large $N$ limit, many $U\left(N^{\prime}\right)$ BPS configurations for $N^{\prime}<N$ are realized in the $U(N)$ theory itself (via reducible configurations), and this may be relevant for the issue of Lorentz invariance in the original BFSS [3] matrix model.

In fact, for $d=3$ one even has a stronger statement. Namely, it is known [9] that the effective gauge group for a BPS configuration is $U\left(N^{\prime}\right)$ where $N^{\prime} \leq N$ is a U-duality invariant function of $N$ and the electric and magnetic quantum numbers. For instance a magnetic flux configuration with $N$ $\mathrm{D} d$-branes and and an $M$-wrapped $\mathrm{D}(d-2)$-brane (we will consider the behaviour of this state under N-duality below), has effectively a $U\left(N^{\prime}\right)$-theory where $N^{\prime}=\operatorname{gcd}(N, M)$ is the greatest common divisor of $N$ and $M$. This can be seen by T-dualizing this to a D1-D1 string system which effectively represents a single D1 string wrapping $N^{\prime}$ times around a particular onecycle of a two-torus.

One can also see explicitly that, via the twisted (toron) boundary conditions, the corresponding BPS state breaks the gauge symmetry down to $U\left(N^{\prime}\right)$. It is in this sense that both the original $U(N)$ configuration with quantum numbers $(N, M)$ and the new $U(M)$ configuration with $\left(N_{\text {new }}=M, M_{\text {new }}=N\right)$ are realized as BPS states in the same, $U\left(N^{\prime}\right)$, gauge theory. If this property persists in some form in higher dimensions, with all the relevant (five-brane, D-brane, ...) quantum numbers included, N-duality could become a true symmetry of the BPS spectrum of 'SYM' theory, at least for large $N$.

\subsection{Problems with $N=0$}

In the above example, after the N-duality we again end up with a configuration containing a top-dimensional brane (and thus permitting a SYM-like interpretation in the standard sense) essentially because, on the M-theory side one is simply exchanging KK momentum quantum numbers in the $d$ 'th and 11'th direction. However, in general this need of course not be the case, as exemplified by the $\mathrm{N}$-dual of the magnetic flux state with quantum numbers $\left(N, m_{12}\right)$ which, according to (4.2) is mapped to a BPS state with zero Dd-brane number, magnetic quantum number $m_{12}$ and electric quantum 
number $e_{d}=N$. Concretely, one has

$$
\begin{array}{ccc}
\text { Example II: } \quad N \mathrm{D} d+m_{12} \mathrm{D}(d-2)_{d} & \stackrel{T^{d-1}}{\longrightarrow} & N \mathrm{D} 1_{d}+m_{12} \mathrm{D} 3_{d} \\
& \stackrel{S_{I I B}}{\longrightarrow} & N \text { F } 1_{d}+m_{12} \mathrm{D} 3_{d} \\
& \stackrel{T^{d-1}}{\longrightarrow} & N \mathrm{~F} 1_{d}+m_{12} \mathrm{D}(d-2)_{d},
\end{array}
$$

Let us stress here that from the present (passive) point of view (permutations of quantum numbers) one is taking the configurations in the first line, mapping them to those in the last line, but reinterpreting them (via an analytic continuation in the parameters $\left(\widetilde{g_{s}}, \ell_{s}, s_{i}\right)$ defining the $\widetilde{\text { II }}$ theory) as configurations in the original $\widetilde{\text { II }}$ theory It is this analytic continuation that is responsible for the fact that U-duality relates BPS states with different energies.

It is also responsible for the fact that, viewed this way, the action of the full U-duality group apparently does not commute with taking the matrix theory limit. In fact, in Example I one is genuinely mapping a $U(N)$ flux state to a $U\left(e_{d}\right)$ flux state, both of which have well-defined finite matrix theory limits (albeit with respect to different backgrounds) and both can be realized as $U\left(N^{\prime}\right)$ configurations where $N^{\prime}=\operatorname{gcd}\left(N, e_{d}\right)$. In Example II, on the other hand, one finds that the well-defined magnetic flux bound state is mapped to a non-threshold bound state with energy

$$
E=\sqrt{\left(\frac{N s_{d}}{\ell_{s}^{2}}\right)^{2}+\left(\frac{m_{12} V_{s}}{g^{2} \ell_{s}^{2} s_{i} s_{j}}\right)^{2}},
$$

whose mass diverges in the $\widetilde{\text { II }}$ matrix theory limit $\ell_{s} \rightarrow 0$ without there being the possibility to subtract a background contribution while retaining a finite result.

The origin of this singular behaviour can be understood in a variety of ways:

1. In the SYM matrix theory picture this behaviour is clearly a consequence of the fact that the N-dual configuration contains no topdimensional wrapped $\mathrm{D} d$-branes which could serve as a background configuration for $(d+1)$-dimensional SYM theory. In fact from the $\mathrm{SYM}_{d+1}$ point of view such configurations, like the above $\mathrm{F} 1-\mathrm{D}(d-2)$ system, are quite singular, describing distributional gauge field configurations where the gauge field is concentrated on a lower-dimensional cycle. In this sense it is not too surprising to find that its energy diverges in the 'SYM' matrix theory limit. 
2. From the IIA string theory point of view, the new $E_{d+1} / E_{d}$ symmetries are associated with T-dualities involving the light-cone direction. In 11] these have been shown to be quite singular in the absence of antisymmetric tensor background fields (see also [30] for an extensive discussion of time-like T-dualities).

3. Finally, from the DLCQ point of view this may also simply be a dual manifestation of the problems with zero light-cone momentum states in the DLCQ (see [31] or [32] for a recent discussion within the present context).

We have not been able to resolve these problems. At first one may have thought that this behaviour is an indication of the fact that the U-duality group of a light-like compactification is smaller than (or a contraction of) $E_{d+1}$, but the results of [22] quoted in [15] suggest that this is not the case. It has already been observed in the past that occasionally string duality requires replacing the traditional gauge theory objects (vector bundles) by something more general. In fact, in [10] it was shown that compatibility of the analysis of 4-2-0 D-brane systems on $T^{4}$ or a K3 with the predictions of string duality can be achieved if one considers moduli spaces of simple coherent semistable (Chan-Paton) sheaves rather than moduli spaces of vector bundles. In this setting, brane configurations with and without top-dimensional branes, 4-branes in this case, can be treated on an equal footing. In particular, 2-0 D-brane systems on a four-torus correspond to sheaves with Mukai vector $\left(0, c h_{1}, c h_{2}\right)$ and these lead to well-defined compact and smooth moduli spaces [33].

While this provides a satisfactory setting for discussing D-brane dualities in $d=4$, it does not generalize immediately in any obvious way to $d \neq 4$ and some new ideas appear to be required. Very recently, the interesting proposal has been put forward by Connes, Douglas and Schwarz [11] and Douglas and Hull [12] that the matrix theory limit of toroidal compactifications with non-trivial background fields along the null direction is described by SYM theory on a non-commmutative torus. It has been argued in [11] that this deformation of SYM theory is necessary if one wants to exhibit the full U-duality group expected from M-theory in the matrix theory.

As here we are mainly dealing with the Weyl subgroup of the U-duality group, non-trivial background fields are not an issue (one can consistently work with a rectangular torus and zero three-form field). Nevertheless, the BPS mass formulae of 11] suggest that within the non-commutative geometry setting also states with $N=0$ have well-defined finite energies for 
a suitable class of modules. This might provide further evidence in favour of the suggestion of [11, 12] that non-commutative geometry is a better arena for matrix theory than SYM on a commutative torus. It also raises the question as to whether there is some relationship, in $d=4$, between non-commutative SYM theory and SYM theory for sheaves.

\subsection{N-Duality, $(1+1)$-Dimensional Backgrounds and Matrix Strings}

We have seen in the previous section that part of the U-duality group is obscured in the matrix theory limit, when one considers the passive action of the U-duality group on the BPS states of a given string theory. On the other hand, as the group $E_{d+1}$ is a manifestation of the general covariance of M-theory compactified on a torus $T^{d+1}$, one expects a realization of the full U-duality group to play a role in establishing the Lorentz invariance of (some future reincarnation of) matrix theory. With this in mind, in this section we shall focus on another realization of the U-duality group, namely in its active sense. In this interpretation, one is explicitly relating states in different string theories (e.g. T-duality changes the radii and exchanges type IIA with type IIB) which have the same energies.

In the context of the first example above, for example, this means that one is now not dealing with a $U\left(e_{d}\right)$ gauge theory in which the $e_{d} \mathrm{D} d$-branes are to be treated as background. Rather, after N-duality it is the image of the $\mathrm{D} d$-brane, i.e. the F1 string, that is to be treated as the background field that becomes infinitely massive in the matrix theory limit. Indeed, the Dd-brane mass in the II theory can be written as

$$
\frac{V_{s}}{g^{2} \ell_{s}^{4}}=\frac{s_{d}}{\ell_{s}^{2} \gamma_{N}}
$$

where $\gamma_{N}$ was defined in (2.14). Comparing with the transformation rules (2.17) one sees that this is precisely the mass of a fundamental string wrapped around the $d$-direction in the $\mathrm{N}$-dual theory. U-duality in the active sense preserves masses and thus the F1 string is the background configuration. This $(1+1)$-dimensional picture is guaranteed by the fact that N-duality not only always maps the top-dimensional D-brane to a wound F1 string (around the $d$-direction), but that also the other N-dual circles shrink to zero size in the matrix theory limit,

$$
s_{i} \rightarrow \frac{\ell_{s}^{2} g^{2} s_{d}}{V_{s}} s_{i} \stackrel{\ell_{s} \rightarrow 0}{\longrightarrow} 0 .
$$


This means that there is always an effectively $(1+1)$-dimensional description of any bound state with a Dd-brane, obtained by Kaluza-Klein reduction. For example in the case of the $\mathrm{D} d$ - $\mathrm{D}(d-2)$-system, with the $(d-2)$-brane wrapping also the $d$-direction, the $\mathrm{D}(d-2)$-brane magnetic flux is represented by a scalar field configuration on the world-sheet of the F1 string, namely

$$
\int \operatorname{tr} F_{i j} \rightarrow \oint \operatorname{tr}\left[X_{i}, X_{j}\right]
$$

where $X_{i}$ are the scalar fields corresponding to the components of the gauge field transverse to the string world-sheet. In fact, more generally, N-duality reproduces precisely the matrix string flux-brane dictionary of 13 in which the D $d$-brane number of the II theory (the D0-brane number of the IIA theory) is represented by an electric flux on the string world-sheet and other configurations correspondingly identified.

\subsection{An M2-Brane Picture of Matrix Theory for $d$ Even}

Let us take a closer look at the matrix string picture we obtain by acting with N-duality on the $\widetilde{\mathrm{II}}$ theory. The N-dual theory is characterized by the string length $\widehat{\ell}_{s}$ and the string coupling constant $\widehat{g}_{s}$. From (2.17,2.18) we have

$$
\begin{aligned}
\widehat{\ell}_{s}^{2} & =\frac{g^{2} \ell_{s}^{4} s_{d}}{V_{s}} \\
\widehat{g}_{s} & =\frac{g^{d-3}}{\ell_{s}^{2} V_{s}^{(d-5) / 2}} .
\end{aligned}
$$

Thus $\widehat{\ell}_{s} \rightarrow 0$ as it should in the matrix theory limit. The string coupling constant $\widehat{g_{s}}$, on the other hand, diverges and thus, as it stands, this matrix string picture is perhaps not the most useful way of describing the situation. At this point a peculiar distinction between $d$ even and $d$ odd arises.2 Namely, for $d$ odd, when one is in a type IIB string theory, one can always perform an S-duality to arrive at a picture in which both the new string length and the string coupling constant tend to zero (as $\ell_{s}^{2}$ ) in the matrix theory limit. For $d=1$ this reproduces exactly the standard $(1+1)$ dimensional SYM matrix theory on the D-string world-sheet. This is true rather trivially as in $d=1 \mathrm{~N}$-duality is the same thing as the $S_{I I B}$-duality. For $d=3$, on the other hand, this should provide a well-defined D-string

\footnotetext{
${ }^{2}$ This was noticed in a discussion with Tom Banks.
} 
description of SYM theory in $(3+1)$-dimensions and it may be interesting to pursue this.

When $d$ is even, this procedure is not available. However, as one is dealing with strongly coupled strings one is motivated to lift this to an $\widehat{M}$-theory characterized by an $\widehat{R_{11}}$ and a new Planck length $\widehat{\ell_{P}}$. This indeed turns out to be a promising thing to do as the length of the new eleventh dimension is

$$
\widehat{R_{11}}=\widehat{g_{s}} \widehat{\ell_{s}}=\frac{g^{d-2} s_{d}^{(d-4) / 2}}{V_{s}^{(d-4) / 2}},
$$

and is therefore constant in the matrix theory limit. Thus for $d$ even the objects that appeared to be strings are actually M-theory membranes. This picture is potentially useful due to the fact that the new Planck length,

$$
{\widehat{\ell_{P}}}^{3}=\widehat{\ell}_{s}^{3} \widehat{g}_{s}=\frac{g^{d} s_{d}^{(d-2) / 2} \ell_{s}^{4}}{V_{s}^{(d-2) / 2}},
$$

goes to zero when $\ell_{s} \rightarrow 0$, implying that the dynamics on the world-volume of this $\widehat{M}$-theory two-brane decouples from the bulk dynamics in this limit. If this description is to be trusted, it should at the very least reproduce the known matrix theory for $d=2$, the $\mathrm{SYM}_{2+1}$-theory on the world-volume of the D2-branes, and this is indeed the case. To see this we note that for $d=2 \widehat{R_{11}}=s_{1}$, and the new radius of the 1 -direction is $\widehat{s_{1}} \sim s_{1} \ell_{s}^{2}$. Thus the $\widehat{M}$ 2-brane becomes a D2-brane in the new IIA string theory associated with shrinking the 1-direction of the $\widehat{M}$-theory, and it is wrapped around the (constant) 2- and 11-directions.

Something more interesting appears to happen for $d=4$. In that case, as we recalled in section 2.3, the matrix theory is actually the world-volume theory on the M5-brane of the $\widetilde{M}$-theory associated to the II string theory [18, 17]. As the M5-brane is the electro-magentic dual of the M2-brane, we would like to suggest that the above description is precisely a dual description of the known matrix theory for $d=4$. In favour of this interpretation, which certainly needs to be substantiated, we note that the parameters of the $\widehat{M}$ and $\widetilde{M}$-theories are related by

$$
\begin{aligned}
\widehat{R_{11}} & =\widehat{\ell}_{s} \widehat{g}_{s}=g^{2}=\widetilde{g_{s}} \ell_{s}=\widetilde{R_{11}} \\
{\widehat{\ell_{P}}}^{3} & ={\widetilde{\ell_{P}}}^{6} \frac{s_{d}}{V_{s}} .
\end{aligned}
$$

This is precisely as required by M-theory 2-brane/5-brane duality which does not change $R_{11}$ but exchanges the M2- and M5-brane tensions $\ell_{P}^{3}$ and $\ell_{P}^{6}$ respectively. 
Naively at least, this membrane picture appears to be valid also for $d=6$, thus prompting the suggestion that this may provide a way of understanding the elusive matrix theory for $d=6$.

Notice that in the $(2,1)$ string construction of M-theory [34], depending upon the choice of null gauging, one finds either the world-volume theory of a Dstring or of a D2-brane as the target space of the $(2,1)$ string worldsheet. These are also the only two possibilities that arise in the constructions described above. In [35] evidence was presented that the $(2,1)$ string theory is related to the maximally compactified matrix theory. Our observation that in all the constructions of lower-dimensional world-volume theories obtained by the action of N-duality on a BPS configuration of the $\widetilde{I I}$ theory one only obtains either string or membrane pictures, just as in the $(2,1)$ string constructions, provides further evidence in support of this conjecture.

\subsection{The Invariant $I$ and Black-Hole Entropy}

Let us briefly come back to the U-duality invariant $I_{d}(2.3,2.12)$ which was important for the algebraic analysis of section 3. For the present purposes we will find it convenient to rewrite this in the compact form

$$
I_{d}^{-1}=\frac{V_{s}}{{\widetilde{g_{s}}}^{2} \ell_{s}^{8}}
$$

valid for any $d$. Although perhaps not immediately obvious at this point, this is precisely the invariant that plays a prominent role in the analysis of supergravity BPS states and black hole entropies (see [26, 36]). In fact, we will now show that for $d=5,6$, corresponding to black holes in five and four dimensions, $I$ coincides with the cubic invariant of $E_{6}$ and the quartic invariant of $E_{7}$ respectively.

In the construction of Strominger and Vafa 37] five-dimensional black holes are labelled by their D5-brane number $Q_{5}$, as well as by the D-string winding number $Q_{1}$ and momentum $P$ in one (and the same) direction. The product of the three dressed charges (fluxes, tensions) is precisely

$$
\frac{Q_{5} V_{s}}{\widetilde{g_{s}} \ell_{s}^{6}} \cdot \frac{Q_{1} s_{6}}{\widetilde{g_{s}} \ell_{s}^{2}} \cdot \frac{P}{s_{6}}=\left(Q_{5} Q_{1} P\right) I_{5}^{-1} .
$$

Alternatively the black holes may be described in the S-dual basis of NS5brane number, string winding and momentum, and of course one finds

$$
\frac{Q_{5} V_{s}}{{\widetilde{g_{s}}}^{2} \ell_{s}^{6}} \cdot \frac{Q_{1} s_{6}}{\ell_{s}^{2}} \cdot \frac{P}{s_{6}}=\left(Q_{5} Q_{1} P\right) I_{5}^{-1},
$$


as guaranteed by the U-duality $E_{6}$-invariance of $I_{5}$.

Four-dimensional black holes, corresponding here to $d=6$, are labelled by four parameters 38] and their entropy is given in terms of a quartic invariant of $E_{7} . I_{6}^{-1}$ has mass-dimension 2, so we expect this quartic invariant to be related to $I_{6}^{-2}$. This is indeed the case. For example, a BPS black hole configuration can be labelled by four D3-brane winding numbers corresponding to D3-branes wrapping the (123), (345), (561), (246) directions. Computing the product of their tensions (as above we could include the corresponding quantum numbers), one finds

$$
\frac{s_{1} s_{2} s_{3}}{\widetilde{g_{s}} \ell_{s}^{4}} \cdot \frac{s_{3} s_{4} s_{5}}{\widetilde{g_{s}} \ell_{s}^{4}} \cdot \frac{s_{5} s_{6} s_{1}}{\widetilde{g_{s}} \ell_{s}^{4}} \cdot \frac{s_{2} s_{4} s_{6}}{\widetilde{g_{s} \ell_{s}^{4}}}=\left(\frac{V_{s}}{{\widetilde{g_{s}}}^{2} \ell_{s}^{8}}\right)^{2}=I_{6}^{-2}
$$

Alternatively, a four-dimensional black hole can be described by the D6brane number and three mutually orthogonal D2-brane winding numbers, leading to the expected result

$$
\frac{V_{s}}{\widetilde{g}_{s} \ell_{s}^{7}} \cdot \frac{V_{s}}{\left(\widetilde{g_{s}} \ell_{s}^{3}\right)^{3}}=I_{6}^{-2} .
$$

This agreement between the U-invariant (4.14) and the invariants appearing in the discussion of black hole entropies is, of course, virtually guaranteed by the paucity of $E_{d+1}$ invariants. But from the traditional point of view the cubic invariant of $E_{6}$ and the quartic invariant of $E_{7}$ appear to be very different objects. (4.14), on the other hand, provides a general and unified expression for the U-duality $E_{d+1}$-invariant for any $d$.

\subsection{A Look at Some States in $d=6$ And $d=7$}

We have already mentioned in section 3.4 that in $d \leq 5$ only one new state, the background $\mathcal{N}$, is needed to unify the momentum and flux $E_{d}$-multiplets into one $E_{d+1}$-multiplet (in the sense of one-particle states). In $d \geq 6$ however, more new states necessarily appear and these should somehow be indicative of the new physics that appears in the matrix theory description of lower-dimensional string compactifications.

For example, for $d=6$, instead of the $27+27=54$ flux and momentum states in the $\overline{\mathbf{2 7}} \oplus \mathbf{2 7}$ of $E_{6}$, we find 56 states in the Weyl group orbit of the $\mathbf{5 6}$ of $E_{7}$. The new state arises by considering the $S L(7, \mathbb{Z}) 7$-plet corresponding to the Taub-NUT TN6-brane on the M/IIA-theory side, with mass

$$
M=\frac{V R_{a}}{\ell_{P}^{9}}, \quad a=1, \ldots, 6,11 .
$$


Here and below $V$ denotes the $(d+1)$-dimensional volume $V=V_{R} R_{11}$. Choosing $R_{a}$ to be one of the spatial circles,say $R_{6}$, one obtains the TN5brane, i.e. the KK monopole, with $R_{6}$ the NUT direction. This turns into the NS5-brane (transverse to $R_{6}$ ) in the $\widetilde{I I}$ theory, with mass

$$
M=\frac{V R_{6}}{\ell_{P}^{9}}=\frac{V_{s}}{s_{6}{\widetilde{g_{s}}}^{2} \ell_{s}^{6}} .
$$

For $R_{a}=R_{11}$, on the other hand, one obtains the D6-brane, thus a D0-brane in the II theory, with mass

$$
M=\frac{V R_{11}}{\ell_{P}^{9}}=\frac{1}{\widetilde{g}_{s} \ell_{s}} .
$$

Clearly these two states are related by $\mathrm{N}$-duality, using $\alpha_{d=6}$, as can also be checked directly by acting with $T_{12345} S_{I I B} T_{12345}$.

The D0-brane does not appear in the SYM flux and momentum multiplets as it cannot form a BPS bound-state with the background D6-branes defining the low-energy SYM theory. In fact, we have to remember that N-duality also acts non-trivially on the background, so not all single-particle states in the orbit of the U-duality group need necessarily be able to form (or appear as) BPS bound states with the background Dd-branes.

However, just using S-dualities (and permutations), one can e.g. map the threshold bound state formed by the background $N$ D6-branes and wrapped D2-branes (instantons) to a threshold bound state consisting of an NS5brane wrapped around the background D6-branes. This state is then of course well-defined in the matrix theory limit (note that (4.20) is constant in that limit as $g^{2}=\widetilde{g}_{s} \ell_{s}^{3}$ in $d=6$ ), and so is therefore its N-dual. As N-duality maps the D6 branes to wrapped NS strings, this N-dual bound state is thus a BPS threshold bound state of D0-branes with $N$ background NS strings wrapped around the 6 -direction, once again providing a $(1+1)$ or $(2+1)$-dimensional picture of this configuration.

Let us now consider $d=7$. Instead of the $56+126=182$ flux and momentum states in the $\mathbf{5 6} \oplus \mathbf{1 3 3}$ of $E_{7}$ we find 240 states in the Weyl group orbit of the $\mathbf{2 4 8}$ of $E_{8}$. Their M-theory masses and those of their II counterparts, together with their $S L(8, \mathbb{Z})$ degeneracy, are displayed in the following table. There $R_{a}, a=1, \ldots, 7,11$ denotes one of the $(d+1)=8$ radii of M-theory, and we have divided the $\widetilde{\text { II }}$ states into longitudinal $(\mathrm{L})$ and transverse $(\mathrm{T})$ states according to whether one of the $R_{a}$ is $R_{11}$ or not (this is for bookkeeping purposes only - it does not mean that these states are necessarily 
longitudinal or transverse on the M-theory side).

\begin{tabular}{|c|c|c|c|}
\hline$S L(8, \mathbb{Z})$ & M-Theory & $\widetilde{\mathrm{II}}(\mathrm{T})$ & $\widetilde{\mathrm{II}}(\mathrm{L})$ \\
\hline 8 & $\frac{1}{R_{a}}$ & $\frac{s_{i}}{\ell_{s}}$ & $\frac{V_{s}}{g^{2} \ell_{s}^{4}}$ \\
\hline 28 & $\frac{R_{a} R_{b}}{\ell_{P}^{3}}$ & $\frac{V_{s}}{g^{2} \ell_{s}^{2} s_{i} s_{j}}$ & $\frac{1}{s_{i}}$ \\
\hline 56 & $\frac{V}{\ell_{P}^{6} R_{a} R_{b} R_{c}}$ & $\frac{s_{i} s_{j} s_{k}}{g^{2}}$ & $\frac{V_{s} s_{i} s_{j}}{g^{4} \ell_{s}^{2}}$ \\
\hline 56 & $\frac{V R_{a}}{\ell_{P}^{9} R_{b}}$ & $\frac{V_{s} s_{i}}{g^{4} s_{j}}$ & $\frac{V_{s}^{2}}{g^{6} \ell_{s}^{2} s_{i}} \& \frac{s_{i} \ell_{s}^{2}}{g^{2}}$ \\
\hline 56 & $\frac{V R_{a} R_{b} R_{c}}{\ell_{P}^{12}}$ & $\frac{V_{s}^{2}}{g^{6} s_{i} s_{j} s_{k}}$ & $\frac{V_{s} \ell_{s}^{2}}{g^{4} s_{i} s_{j}}$ \\
\hline 28 & $\frac{V^{2}}{R_{a} R_{b} \ell_{P}^{15}}$ & $\frac{V_{s} \ell_{s}^{2} s_{i} s_{j}}{g^{6}}$ & $\frac{V_{s}^{2} s_{i}}{g^{8}}$ \\
\hline 8 & $\frac{V^{2} R_{a}}{\ell_{P}^{18}}$ & $\frac{V_{s}^{2} \ell_{s}^{2}}{g^{8} s_{i}}$ & $\frac{V_{s} \ell_{s}^{4}}{g^{6}}$ \\
\hline
\end{tabular}

As little (or practically nothing) appears to be known about matrix theory on $T^{7}$ (but see [15]), a concrete (or even tentative) identification of some of the states on the 'SYM' side will be difficult to come by. However, let us make a few comments on this U-duality spectrum:

1. To understand at least in part, some of the new states appearing in this table, recall that carrying out a T-duality on a circle transverse to the world volume of the NS5-brane produces a TN5-brane and vice versa. Once we compactify on a 7-torus the NS5-brane has two transverse directions and we can apply two independent transverse T-dualities. From the NS5-brane of the $\widetilde{\mathrm{II}}$ theory, two T-dualities produce the configuration with mass $\frac{V_{s} s_{i} s_{j}}{g^{4} \ell_{s}^{2}}$.

2. The states in this table that really require some interpretation however are not these, but rather those with a mass behaving as inverse powers of $g^{2}$ or $\widetilde{g_{s}}$ greater than 2 . For instance applying N-duality to the nonNUT transverse direction of the TN5-brane, one obtains a 'rolled-up' version of the TN6-brane, corresponding to the first set of longitudinal states in the fourth row. Such a configuration has been considered (in a very different context) in [14 and will be discussed in more detail in the next section. Note that this latter set of states displays the unusual $1 / g_{s}^{3}$-dependence on the IIA/M-theory side noted in [2]. The $\widetilde{I I}$-counterpart of this rolled-up TN6-brane, which displays a $1 / \widetilde{g}_{s}{ }^{3}$ behaviour, can form a half-BPS bound state with the background D7branes with energy $E_{S Y M}=V_{s}^{3} / N g^{10} s_{i}^{2}$. Note that this is just one of many states in the table that have this peculiar dependence on the coupling constants. 
3. In total the new states we find are the longitudinal state in the first row, seven longitudinal states in the fourth row (last entry), twentyone longitudinal states in the fifth row, twenty-one transverse states in the sixth row, and finally all of the eight states in the last row.

4. The new states in the fourth row can be concretely identified as wound D1-strings since $s_{i} \ell_{s}^{2} / g^{2}=s_{i} / \widetilde{g}_{s} \ell_{s}^{2}$. Although of course wound D1strings can appear in every odd dimension, these play a special role here for the same reason that D0-branes are special in $d=6$ : they cannot form BPS bound states with the background $\mathrm{D} d$-branes and thus they can only appear after a transformation like N-duality that acts non-trivially on the background.

\subsection{A Rolled-Up Taub-NUT Soliton And $1 / g_{s}^{3}$-States}

For compactifications with $d \geq 7$, it was observed in [2] that in the U-duality multiplet one finds states which in the IIA string theory picture have masses that behave like $1 / g_{s}^{3}$ or higher inverse powers of $g_{s}$. These are clearly neither D-brane nor solitonic p-brane objects. For objects of this type it was argued in [2] that the gravitational field will be very large - in particular they will not correspond to space-times that are asymptotically flat. One can actually construct an explicit example of such a metric by considering for $d=7$ the states with mass

$$
M=\frac{R_{a}^{2} R_{b_{1}} \ldots R_{b_{6}}}{l_{P}^{9}},
$$

This is the Taub-NUT 6-brane of M-theory. Reaching the IIA string by compactifying on the $R_{a}$ circle leads to the D6-brane with mass proportional to the product of the 6 radii divided by $g_{s} l_{s}^{7}$; compactifying on one of the $R_{b_{i}}$ we find the TN5-brane of IIA string theory, with mass proportional to a product of radii (with one squared as for the TN6-brane) divided by $g_{s}^{2} l_{s}^{8}$. However, if we compactify on the remaining one of the $d+1=8$ circles of M-theory we obtain an object with mass

$$
M=\frac{R_{a}^{2} R_{b_{1}} \ldots R_{b_{6}}}{g_{s}^{3} l_{s}^{9}} .
$$

Given that we started with a TN6-brane, this compactification clearly corresponds to taking an infinite array of TN6-branes and compactifying along this periodic direction. 
The multi-TN metric has the form

$$
\begin{aligned}
d s^{2} & =V^{-1}(d \psi+\vec{A} \cdot d \vec{x})^{2}+V d \vec{x}^{2} \\
V & =\frac{1}{L^{2}}+\frac{1}{2} \sum_{i=1}^{k} \frac{\ell_{P}}{\left|\vec{x}-\vec{x}_{i}\right|},
\end{aligned}
$$

$\psi \in\left[0,2 \pi \ell_{P}\right], \vec{x}=(x, y, z)$. The length of the circle at $\infty$ is $2 \pi \ell_{P} L$.

Now consider a configuration with $\vec{x}_{i}=(i a, 0,0)$ for $i \in \mathbb{Z}$. Note that the sum in $V$ now diverges, but subtracting an infinite constant we can perform the sum using Poisson resummation. The result is a new metric with $V$ given by,

$$
V(x)=\frac{1}{2 \pi} \log \left(\frac{\mu a}{\rho}\right)+\sum_{m \neq 0} \mathrm{e}^{2 \pi i m \frac{x}{a}} K_{0}\left(\frac{2 \pi|m| \rho}{\lambda a}\right),
$$

where $\mu$ is some constant and $\rho=\sqrt{y^{2}+z^{2}}$. The new metric is periodic in $x$ and $\psi$ and choosing the $R_{11}$ direction to correspond to $x$ we find the metric corresponding to the state with mass proportional to $1 / g_{s}^{3}$. It is amusing to notice that precisely this metric arises in the analysis of the hypermutiplet moduli space of the IIA string theory in the vicinity of the conifold singularity [14]. Furthermore, due to the explicit appearance of the logarithmic term in $\mathrm{V}$, the metric is not asymptotically flat but log-divergent, as predicted by the general arguments of [39].

In $d=7$ as we saw in the previous subsection, there are many states of this type with mass proportional to higher inverse powers of $g$, E.g. the M-theory mass of the states in the fifth row is,

$$
M=\frac{R_{a}^{2} R_{b}^{2} R_{c}^{2} R_{d_{1}} R_{d_{2}} R_{d_{3}} R_{d_{4}} R_{d_{5}}}{\ell_{P}^{12}} .
$$

This seems to require some $5 d$ Euclidean configuration (gravitational instanton) with three NUT directions, generalizing the Euclidean Taub-NUT solution in $d=4$. Such an object appears not to be known. U-duality, however, would predict that it can form a threshold bound state with D7branes.

\section{Acknowledgements}

We are grateful to Tom Banks, Fawad Hassan, K.S. Narain and George Thompson for useful discussions at various stages of this work. This work was supported in part by the EC under the TMR contract ERBFMRXCT96-0090. 


\section{REFERENCES}

[1] C. Hull and P. Townsend, "Unity of Superstring dualities", Nucl.Phys. B438 (1995) 109, hep-th/9410167.

[2] S. Elitzur, A. Giveon, D. Kutasov and E. Rabinovici, "Algebraic aspects of matrix theory on $T^{d "}$, hep-th/9707217.

[3] T. Banks, W. Fischler, S. Shenker and L. Susskind, "M Theory as a Matrix Model", Phys. Rev. D55 (1997), 5112, hep-th/9610043.

[4] T. Banks, "Matrix Theory", hep-th/9710231.

[5] A. Sen, "D0-branes on $T^{N}$ and matrix theory", hep-th/9709220.

[6] N. Seiberg, "Why is the matrix model correct?", hep-th/9710009.

[7] L. Susskind, "Another conjecture about M(atrix) theory", hep-th/9704080.

[8] W. Nahm, "Monopoles in quantum theory", Proceedings of the Monopole Meeting, Trieste, eds. Craigie et al; W. Nahm, "Self-dual monopoles and calorons", Lecture notes in Physics 201, Springer-Verlag, (1984); E. Corrigan and P. Goddard, "Construction of instanton and monopole solutions and reciprocity", Ann. Phys. 154 (1984) 253; P.J. Braam and P. van Raal, "Nahm's transformation for instantons", Nucl. Phys. B460 (1989) 267.

[9] F. Hacquebord and H. Verlinde, "Duality Symmetry of $N=4$ Yang-Mills theory on $T^{3}$ ", hep-th/9707179.

[10] J. Harvey and G. Moore, "On the algebras of BPS states", hep-th/9609017.

[11] A. Connes, M.R. Douglas and A. Schwarz, "Noncommutative Geometry and Matrix Theory: Compactification on Tori", hep-th/9711162

[12] M.R. Douglas and C.M. Hull, "D-branes and the Noncommutative Torus", hep-th/9711165

[13] R. Dijkgraaf, E. Verlinde and H. Verlinde, "Matrix string theory", Nucl.Phys. B500 (1997) 43, hep-th/9703030; "Notes on matrix and micro strings", hep-th/9709107.

[14] H. Ooguri and C. Vafa, "Summing up D-instantons", Phys.Rev.Lett. 77 (1996) 3296, hep-th/9608079; N. Seiberg and S. Shenker, "Hypermutiplet moduli space and string compactification to three-dimensions", Phys.Lett. B388 (1996) 521, hep-th/9608086.

[15] C.M. Hull, "Matrix Theory, U-Duality and Toroidal Compactification of MTheory", hep-th/9711179

[16] C.M. Hull (to appear)

[17] M. Berkooz, M. Rozali and N. Seiberg, "Matrix description of M-theory on $T^{4}$ and $T^{5 ",}$ hep-th/9704089; N. Seiberg, "Matrix description of M-theory on $T^{5}$ and $T^{5} / Z_{2}$, hep-th/9705221. 
[18] M. Rozali, "Matrix theory and U duality in seven-dimensions", Phys.Lett. B400 (1997) 260, hep-th/9702136.

[19] D. Berenstein, R. Corrado and J. Distler, "On the moduli spaces of M(atrix) theory compactifications", hep-th/9704087.

[20] W. Taylor, "D-brane field theory on compact spaces", Phys. Lett. B394 (1997) 283 , hep-th/9611042.

[21] R. Dijkgraaf, E. Verlinde and H. Verlinde, "BPS quantization of the fivebrane", Nucl. Phys B486 (1997) 89, hep-th/9604055.

[22] C. Hull and B. Julia (to appear)

[23] J. Maldacena, "Branes probing black holes", hep-th/9709099.

[24] A. Hanany and G. Lifschytz, "M(atrix) theory on $T^{6}$ and a M(atrix) theory description of KK monopoles", hep-th/9708037; A. Losev, G. Moore and S. Shatashvili, "M \& m's", hep-th/9707250; I. Brunner and A. Karch, "Matrix description of M-theory on $T^{6 ",}$, hep-th/9707259; O. Ganor, "On the M(atrix) model for M-theory on $T^{6}$ ", hep-th/9709139.

[25] R.W. Gebert and H. Nicolai, "E10 for beginners", hep-th/9411188.

[26] S. Ferrara and J. Maldacena, "Branes, central charges and U-duality invariant BPS conditions", hep-th/9706097.

[27] E. Bergshoeff and M. de Roo, "D-branes and T-duality", Phys, Lett. B380 (1996) 265, hep-th/9603123, M. Green, C. Hull and P. Townsend, "D-brane Wess-Zumino actions, T-duality and the cosmological constant", Phys. Lett. B382 (1996) 65, hep-th/9604119.

[28] M. Douglas and G. Moore, "D-branes, quivers, and ALE instantons", hep-th/9603167.

[29] C. Vafa, "Instantons on D-branes", hep-th/0512078; Z. Guralnik and S. Ramgoolam, "Torons and D-brane bound states", hep-th/9702099, "From 0-branes to torons", hep-th/9708089; R. Gopakumar, "BPS states in matrix strings", hep-th/9704030.

[30] G. Moore, "Finite in all directions", hep-th/9305139.

[31] M. Burkhardt, "Light Front Quantization", hep-th/9505259

[32] S. Hellerman and J. Polchinski, "Compactification in the Lightlike Limit", hep-th/9711037.

[33] S. Mukai, "Symplectic structure of the moduli space of sheaves on an abelian or K3 surface", Invent.Math. 77 (1984) 101.

[34] D. Kutasov and E. Martinec, "M-branes and $N=2$ strings", Class. Quant. Grav. 14 (1997), 2483, hep-th/9612102.

[35] E. Martinec, "M-theory and $N=2$ strings", hep-th/9710122. 
[36] G. Horowitz, J. Maldacena and A. Strominger, "Nonextremal Black Hole Microstates and U-duality", Phys. Lett. 383 (1996) 151, hep-th/9603109; R. Kallosh and B. Kol, "E(7) Symmetric Area of the Black Hole Horizon", Phys. Rev. D53 (1996) 5344, hep-th/9602014; S. Ferrara and R. Kallosh, "Universality of Supersymmetric Attractors", Phys. Rev. D54 (1996) 1525, hep-th/9603090.

[37] A. Strominger and C. Vafa, "Microscopic origin of the Bekenstein-Hawking Entropy", Phys. Lett. B379 (1996), 99, hep-th/9601029.

[38] J. Maldacena and A. Strominger, "Statistical Entropy of Four-dimensional Extremal Black Holes", Phys. Rev. Lett. 77 (1996), 428, hep-th/9603060.

[39] T. Banks and L. Susskind, "The number of states of two-dimensional critical string theory", Phys.Rev. D54 (1996) 1677, hep-th/9511193. 